\title{
Dark Energy and its Cosmic Density from Einstein's Relativity and Gauge Fields Renormalization Leading to the Possibility of a New 'tHooft Quasi Particle
}

\author{
Mohamed S. El Naschie* \\ Department of Physics, University of Alexandria, Egypt
}

\begin{abstract}
Since in Euclidean and Riemannian continuous smooth geometry a point cannot rotate, it follows then that only a finite length line could rotate. Overlooking this simple evident and trivial point is the cause of most of the troubles associated with the general theory of relativity. Once realized, the situation could be resolved by going in the directions of Cartan-Einstein spacetime but all the way without wavering. The present work which represents also a short survey on the subject combines the mental picture afforded by Cosserat micro-polar spacetime with that of Cartan-Einstein spacetime as well as the Cantorian-fractal spacetime proposal. In the course of doing that we resolve the major problem of dark energy. Various methods are used to validate our main results including 'tHooft-Veltman renormalization method. In particular the 'tHooft -Veltman-Wilson scheme suggests the possibility of two new exotic quasi-particles stemming from the fractal nature of quantum spacetime which resembles a transfinite cellular automata relevant to Auffray's xonic quantum physics.
\end{abstract}

Keywords: Cosserat's spacetime, cartan-Einstein's proposal cosmic expansion, dimensional regularization, dark energy, exotic quasi particle, fractal-Cantorian spacetime, modified relativity, meta energy, pure gravity, relativistic theory of elasticity, rindler spacetime, 'tHooft renormalon, transfinite cellular automata, xonic quantum physics.

\section{GENERAL INTRODUCTION}

For the author, an engineer by profession with Applied Mechanics with training and professional work in high energy physics and cosmology are the most fascinating subjects in science [1-6]. The present work is both a state-of-the-art account of the fractal-Cantorian spacetime proposal in high energy physics and cosmology as well as being partially an original contribution to the subject announcing various new results and elementary particles [1-164].

Let us start with a classical elasto-mechanical model which displays a remarkable quasi-nonclassical behavior. Elastic cylindrical shells $[7,6,25,62,113,114]$ when pinched in the middle deform in the following slightly unexpected way: Locally, in the vicinity of the pinching [149], the circular cross-section deforms to an oval shaped one. With increasing distance from the pinching region the oval cross-section rotates until it becomes perpendicular to the oval at the pinched middle of the cylinder (see Fig. 1). This kind of deformation is only possible because of the 'material' nature of the cylinder and is a consequence of the continuum mechanics of a tangible material surface as opposed to an idealized purely geometrical non-materialistic space like those theoreticized by Euclid and Riemann [90-93]. Cylindrical shells are real structures and as such are endowed with complex shear and torsional forces as is well known from the

*Address correspondence to this author at the Department of Physics, University of Alexandria, Egypt; Tel: 0020100100 9770;

E-mail: chaossf@aol.com theory of elasticity, plasticity and rheology $[25,60,62,78$, $113,114]$. Likening the local curvature in the pinched region with a positive attractive gravity pulling things together we are logically justified to liken the perpendicular curvature at the extremity of the cylinder with a negative repulsive gravity that pushes things apart $[126,128,153]$.

The present work is concerned exclusively with theoretical physics and cosmology of space, time, matter as well as the quanta of ordinary energy [1-8] and of Meta energy, i.e. dark energy [9-28]. The simple analogy between gravity and the deformation of an elastic shell outlined above is taken literally and pushed to its ultimate by imaging the whole set up taking place in four dimensional space akin to that of Einstein's general relativity but with some additional elements due to Cosserat and Cartan as well as $\mathrm{f}(\mathrm{T})$ gravity, pure gravity, Rindler spacetime, relativistic hydrodynamics, elasticity, plasticity and transfinite E-infinity Cantorian spacetime [29116]. To tame the involved 'infinitely' long 4D 'quasi cylinder' we use the sophistication of hyperbolic geometry and utilize the Poincare-Beltrami projection to establish a connection to a Penrose-like fractal tiling universe which represents an effective fiber bundle theory as discussed by Mukhamedov [7-9, 29-77]. It is then not particularly difficult to imagine what one will discover next when connecting each of the ramified fractal tiles to a hyperbolic fractal Rindler space (see Fig. 2). At the circular horizon of the Poincare-Beltrami projection and taking the isomorphic length into consideration, each fractal point is a head of a Rindler wedge $[9,17,29]$. In turn the wedge consists of two parts, a 


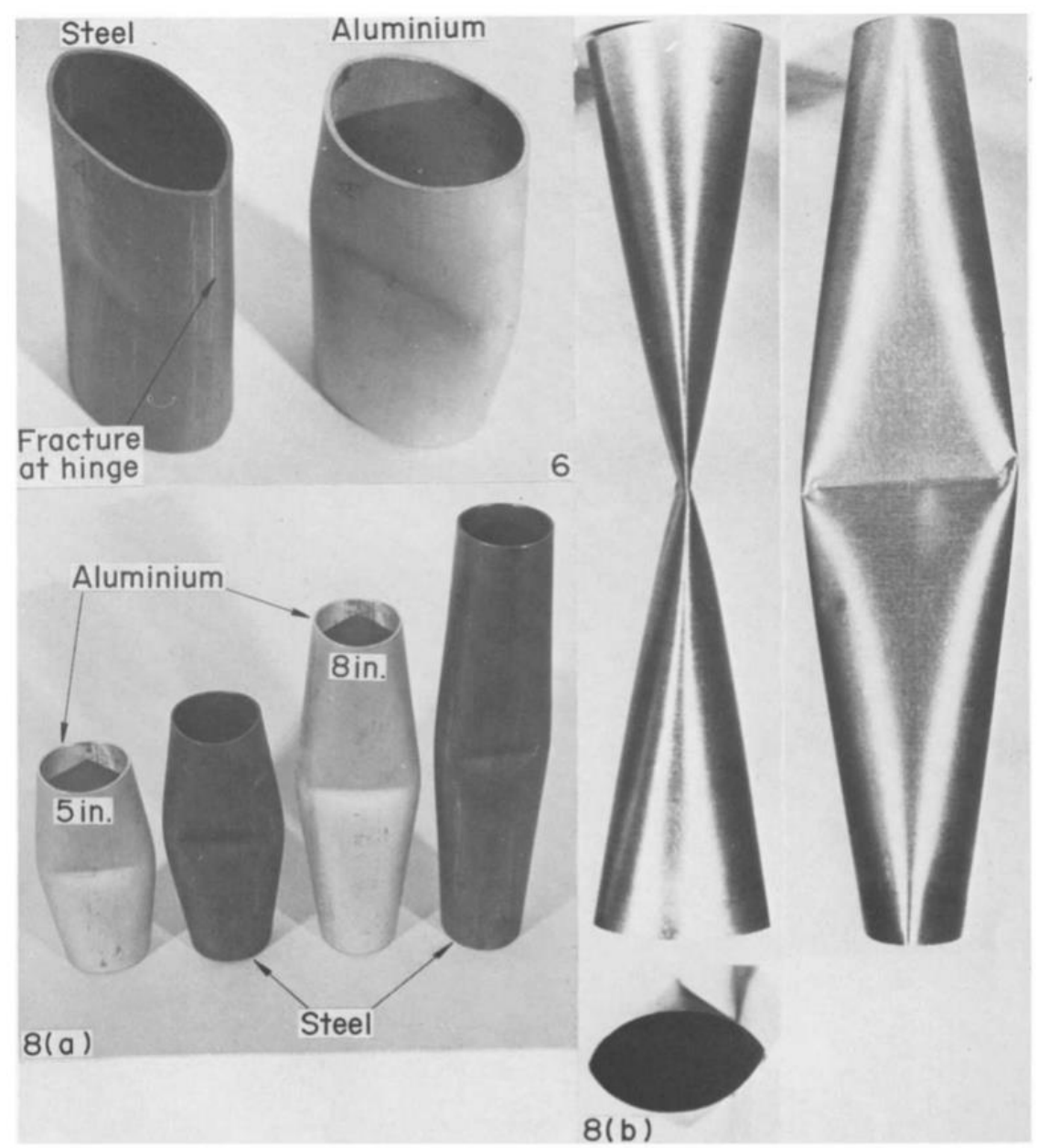

Fig. (1). Actual experiments with elastic and plastic cylindrical shells [74]. The deformation of the pinched cylinder provide an instructive insight into the difference between real material space and abstract mathematical idealization of space. In a real space a local change of curvature at the middle of the pinched cylinder induces a seemingly opposite change of curvature at the edges of the shells. We imagine the situation in $4 \mathrm{D}$ space to be a higher dimensional analogue to gravity and anti-gravity.

hyperbolic triangle with a 'topological' area equal to $\phi^{5} / 2$ where $\phi=(\sqrt{5}-1) / 2$ and a circular segment shape joined to the triangle with an area or rather a topological measure equal to $1-\left(\phi^{5} / 2\right)=5 \phi^{2} / 2[9,17,29]$. Subsequently we use various facts connected to the thermodynamical interpretation of gravity [33], Hawking's radiation [3, 15, 36, 51], noncommutative geometry, Cantorian E-infinity theory as well as the algebraic topology theory of cosmic defects [41] to reason that $\phi^{5} / 2$ which corresponds to a five dimensional zero point stems from three field theoretical dimensions of pure gravity, as given by the vierbien representation $\mathrm{D}=\mathrm{d}(\mathrm{d}-3) / 2$ [125]. This gives us the COBE, WMAP and Planck measured $4.5 \%$ energy of the cosmos $\mathrm{E}(0)=\left(\phi^{5} / 2\right.$ ) $\left(\mathrm{mc}^{2}\right) ; \mathrm{mc}^{2} / 22$ while $5 \phi^{2} / 2$ corresponds to a five dimensional empty set stemming from two field theoretical dimensions of pure gravity. On the other hand the $95.5 \%$ factor of the 'missing' dark energy density given by the said 5-D empty set $\mathrm{E}(\mathrm{D})=\left(5 \phi^{2} / 2\right)\left(\mathrm{mc}^{2}\right)$ corresponds to the antigravity effect behind the observed accelerated expansion of the cosmos $[7,9,20,23]$. In this sense and by setting space, time and matter truly on the very same footing we could loosely say that attractive gravity pinches the 'material' spacetime counterpart of Einstein's gravity and produces the observed puzzling anti-gravity accelerating expansion of the cosmos [36]. In fact it is natural to have negative curvature and thus negative gravity in a Cantorian-fractal spacetime where there are no real points at all and therefore torsion does not vanish by taking the deceptive limit of a fundamentally granular spacetime setting [125, 149]. We conclude this thread by noting that a cosmological constant $\Lambda=-1$, a topological empty set dimension $\mathrm{D}_{\mathrm{T}}=-1$, negative curvature at a horizon or a conjectured negative dimension of a texturetopological defect (see Table $\mathbf{1}$ of section 9) as well as a field theoretical degree of freedom equal -1 for pure 2D gravity are all but basically tautological statements saying essentially the same thing, namely that there is a cosmic accelerated expansion and that dark energy is what stands behind this negative gravity force [69]. In other words we have various mental pictures and different mathematical formulations however it is still the same empirical reality.

A universal wisdom that has been well tested over the years is that in science as in life, asking the right question is 
Table 1. Cosmic topological defects following the classification of Vilenkin and Shellard [52]. Note that we added the conjecture dim(textures) $=-1$ which in effect equates texture to an empty Cantor set.

\begin{tabular}{|c|c|}
\hline Topological Defect & Dimension \\
\hline \hline Domain walls & 2 \\
\hline Strings & 1 \\
\hline Monopoles & 0 \\
\hline Textures & -1 (conjectured) \\
\hline
\end{tabular}

almost half of the answer [1]. It seems that a few scientists were more equipped to ask the right question than most of us and this is the main philosophy probing the present paper [114]. Hermann Weyl's famous book "Raum, Zeit, Materie" may be as good a starting point as any [2]. Do we really treat space, time and matter in a democratic way? Sure enough space and time were fused by the Murkowski-Einstein program however nothing similar was systematically undertaken with the same vigor regarding matter and a somewhat naive materialism prevails in physics [3-5]. For instance the geometry of spacetime used in physics is nowhere taken to be as "real" as the geometry used in say the theory of elasticity or plasticity $[25,60,62,113]$. Of course there are many models used in relativistic quantum physics which utilized hydrodynamical paradigms and even modified fluid mechanics equations but these important efforts are relatively the exception and do not go as far as one could imagine [24]. To achieve our goal, i.e. to put spacetime and matter on the same footing requires a new material-like geometry [6-28] with granular structure for which the torsional part of the connection [24] does not vanish. Thus finding this materiallike geometry [25] is paramount.

In the present work we advocate among other things the idea that such geometry exists since a relatively long time and that it is a generalization of what E. Cartan [101, 102] and the brothers Cosserat developed in 1909 [78] when married to modern Cantorian fractals [7, 8, 12, 14]. In fact we will show various completely unsuspected relations between metal forming engineering problems and the negative pressure behind the observed unexpected acceleration rather than deceleration of cosmic expansion [15-17]. Said succinctly in a few sentences, we will show that anti-gravity is essentially the same phenomena as anti-curvature of a pinched long cylindrical shell once this cylinder is put in the projective hyperbolic plane corresponding to 4 and 5 dimensional fractal spacetime $[7,8,12,14,66]$. Incredible as it may seem at first sight, this is essentially the same thing as saying that dark energy comes from pure gravity as well as the equivalent massless graviton field theoretical $D=d(d-3) / 2$ degrees of freedom where $d$ is the dimension of the space of gravity which are in turn related to Weyl tensor and the empty set in 5 dimensional Kaluza-Klein spacetime as well as the representation of the Vierbien discussed earlier on. The present work is thus a monolithic synthesis of the work of Einstein, Cosserat, Cartan, Hawking, Rindler, Penrose, Conne,
Unruh, 'tHooft and the school of fractal Cantorian spacetime to mention only a few of the main sources pouring into the present work [15-78]. In addition we will look carefully at the role of the killing-Yang tensor in explaining negative energy all apart of an instructive analogy between dark energy and capillary forces of hydrodynamics as well as Koiter's theory of imperfection sensitivity of elastically buckled shells $[25,112,113]$. In the final part of this paper in addition to drawing analogies between our Cantorian spacetime model and infinite time transfinite Cellular Automata [158161], our main results are validated using an extended 'tHooft-Veltman renormalization [119, 120] and two new quasi particles namely Entangelon and 'tHooft renormalon i.e.'tHoofton are conjectured. A readable mathematical introduction to the present work is a recent paper by Auffray [164] which outlines highly interesting connections to his xonic quantum physics. An equally highly recommended popular but accurate introduction to the present work are the six articles by Dr. Mae-Wan Ho in the notable scientific magazine 'Science in Society' [139, 140].

\section{KEEPING AN OPEN MIND ABOUT THE FRAC- TAL-THERMODYNAMICAL FLUCTUATION ORI- GIN OF GRAVITY}

It is important that we point out from the outset a few fundamental points which represent some departure from the orthodoxy of general relativity. In short this requires what we consider a minimum of liberal open mindedness about the following admittedly not universally accepted concepts and experimental findings:

1. As in Feynman's conjecture extended by the author we will occasionally view gravity as the effect of the passing of fractal time [7, 8, 21, 22].

2. We tend to accept that Hawking's radiation, Rindler's wedge and Unruh's temperature are backed by real physics and are by no means mathematical artifacts $[9,17]$.

3. We are firm on the opinion that Hardy's quantum entanglement is real and was experimentally verified. The golden mean to the power of five first found by Hardy as a quantum probability and recognized as such by the author is profound [19].

4. The COBE, WMAP and Planck measurements as well as other recent astronomical as well as astrophysical anomalies are real $[47,80]$ and will not be dismissed here as misinterpretation, faulty calculations or defects of electronic equipment. This is definitely a majority view of scientists worldwide although I must hasten to say that scientific facts have nothing to do with democratic elections.

5. The field theoretical concept of the number of degrees of freedom for pure gravity is of fundamental mathematical and physical importance and is related to dimensionality of the zero set, the empty set as well as to the density of dark energy via $\mathrm{D}=\mathrm{d}(\mathrm{d}-3) / 2$ of the Vierbien and the massless graviton where $d$ is the dimension [99]. Inserting in $D$ it becomes evident that $\mathrm{D}=-1$ for $\mathrm{d}=2$ is a quasi empty set. Now the degrees of freedom of a massless graviton are also 
given by the same formula showing quantum mechanics at the root of classical relativity [27, 70,99].

6. Dimensional regularization $D-4=\epsilon$ is essentially going into the direction of an effective quantum gravity theory and setting $\in=\mathrm{k}=2 \phi^{5}$ leads directly to the exact dark energy density $E(D)=\gamma(D) m^{2}$ where $\gamma(D)=(4-k) / 4=$ $\phi^{5} / 2 ;(21 / 22)[119,120]$. In fact we are tempted to conjecture the existence of two quasi particles given by $\phi^{5}$ and $2 \phi^{5}$ based on this method.

7. 'tHooft's dimensional renormalization method is tacitly a statement on the fractal nature of spacetime and implies that gravity correction to the running coupling constants of four dimensional gauge forces interaction can be substantial at both the Planck scale and by duality the cosmic Hubble scale. This is obvious from $\mathrm{E}(\mathrm{D})=[(4-\mathrm{k}) / 4] \mathrm{mc}^{2} ; \mathrm{mc}^{2}$ (21/22) [119, 120].

8. Cantor sets and the associated golden mean arithmetic are a form of realizing infinite time transfinite Cellular Automata $[18,158-161]$. At this point it is appropriate to note the work of Padmanabhan $[32,33]$ as one of the main guiding lights in uncovering the thermo-dynamical roots of gravity. On the other hand our hyperbolic geometrical fractal conception of spacetime [79] is also at the root of thermodynamics itself as is obvious from the thermal character of Unruh's temperature $[9,17,27]$. The same viewpoint applies of course to electromagnetism where we are justified in seeing $\bar{\alpha}_{o} \cong 137$ as by far more fundamental than Newton's constant, the speed of light or Planck's constant. Finally severe discrepancy between measurement and theory is nothing new and is well documented in all situations where the environment is highly unstable such as is the case with the imperfection sensitivity of buckling of elastic shells [112,113] which was incidentally the Ph.D. thesis of the Author and based upon the work of the leading near to legendary Dutch engineering scientist, W.T. Koiter [112, 113].

\section{EINSTEIN IN COSSERAT-CARTAN SPACE}

The aim of the present section is to demonstrate how easy it is to reformulate and rephrase Einstein's general relativity within the frame work of the theories of Cosserat [78], Cartan and Yano [100-103] to account for the observed and quite surprising accelerated cosmological expansion of the universe and the concurrent inference that almost $95.5 \%$ of the total energy density of the universe seems to be negative dark energy [10-27].

We start from the premise that both Einstein's spacetime and the maximally symmetric Witten's five Branes model leads to the same Lorentzian factor $\gamma=1$ for the maximal Einstein energy density, $\mathrm{E}=\gamma \mathrm{mc}^{2}$ where $\mathrm{m}$ is the mass and $\mathrm{c}$ is the speed of light and will look upon Cartan's affine connection from a Lie symmetry groups view point [96-98]. Never the less the trivial identity:

$\gamma=\mathrm{D}^{(4)} / \mathrm{D}^{(4)}=\mathrm{N}_{\mathrm{K}}^{(32)} / \mathrm{N}_{\mathrm{K}}^{(32)}=1$. where $\gamma$ is the Lorentz factor, $\mathrm{D}^{(4)}=4$ and $\mathrm{N}_{\mathrm{K}}{ }^{(32)}=$ (32)(33) $/ 2=528$, implies a far more intricate relation than the deceptively harmless appearance transpires. The rationale behind this assertion is that exactly 504 of the 528 particlelike quantum states may be at least heuristically identified as Cartan-like torsional states [90-93]. This could be deduced with relative ease from an educated counting exercise of the quantum states of Heterotic string theory [103]. In the course of doing that it will become clear that the 504 are the internal killing-Yano hidden dimensions of E8E8 exceptional Lie symmetry group of superstrings plus 8 [106-108]:

$$
\begin{aligned}
& \mathrm{D}^{(8)}+\operatorname{dim} \mathrm{E} 8 \mathrm{E} 8=8+|\mathrm{SO}(32)|=8+(2)(248)=8+496 \\
& =504 .
\end{aligned}
$$

Details of the computation and counting are given lucidly on pages 383-385 of Ref. [103]. The 528 killing vector fields on the other hand are interpreted by us here in two ways. First it is the number of components of the killing-Yano conformal tensor [25] and second it is the sum of the dimensions of E8, E7, E6, E5 and E4 [10-12]. Based on its Dykin diagram E5 is just another name for $|\mathrm{SO}(10)|=(10)(9) / 2=45$. In other words we have [106-108]:

$$
\begin{aligned}
& \sum_{i=5}^{i=8}\left|E_{i}\right|=\left|E_{5}\right|+\left|E_{6}\right|+\left|E_{7}\right|+\left|E_{8}\right|=45+78+133+248 \\
& =504 .
\end{aligned}
$$

Adding $|\mathrm{E} 4|=24$ where $|\mathrm{E} 4|$ is simply another name for $|\mathrm{SU}(5)|$ of GUT unification $[104,105]$, we see that:

$\sum_{\mathrm{i}=4}^{\mathrm{i}=8}\left|\mathrm{E}_{\mathrm{i}}\right|=504+\mid \mathrm{SU}(5)=504+\left[(5)^{2}-1\right]=504+24$

$=528$.

In other words we have [106-108]:

$\sum_{\mathrm{i}=4}^{\mathrm{i}=8}\left|\mathrm{E}_{\mathrm{i}}\right|=\mathrm{N}_{\mathrm{K}}^{(32)}=528$.

Consequently the number of the killing components [84] which are related to the purely "ordinary" energy are given by:

$\mathrm{N}_{\mathrm{K}}^{(32)}-\sum_{\mathrm{i}=5}^{\mathrm{i}=8}\left|\mathrm{E}_{\mathrm{i}}\right|=\sum_{\mathrm{i}=4}^{\mathrm{i}=8}\left|\mathrm{E}_{\mathrm{i}}\right|-\sum_{\mathrm{i}=5}^{\mathrm{i}=8}\left|\mathrm{E}_{\mathrm{i}}\right|=528-504=24$

$=\operatorname{dim} \mathrm{SU}(5)$.

It is vital at this point not to confuse dark energy with torsion energy due to the 24 Riemann-Cartan connection components in four dimensions because the concept torsion has various meanings in string theory compared to EinsteinCartan theory $[102,103]$. In the present work the $4.5 \%$ cosmically measured ordinary energy density is due to the 24 Yano-killing tensor components while the conjectured dark energy is due to the 504 rest components known from the spectrum of Heterotic string theory [103].

Two further relevant observations regarding the vital number 24. First it is exactly equal to the number of the gauge bosons of SU(5) GUT unification which adds 12 bos- 
ons to the well known and experimentally found 12 bosons of the SU(3) SU(2) U(1) standard model of high energy physics [106-108]. Second the only pure number in the killing-Yano totally skew symmetric tensor of the well known 5 expression is $\mathrm{e}_{\sigma \pi \in \lambda}$ e $\sigma \pi \in \lambda=-24$.

Contemplating the situation a little it is not particularly difficult to convince oneself that the Lorentzian factor of Einstein's energy density corresponding to 24 non-trivial Bianchi identities is the ratio between the 24 and the Witten bulk of 528 maximally symmetric space. Consequently ordinary energy comes with a Lorentzian factor:

$$
\gamma_{\mathrm{o}}=\frac{\sum_{\mathrm{i}=4}^{\mathrm{i}=8}\left|\mathrm{E}_{\mathrm{i}}\right|-\sum_{\mathrm{i}=5}^{\mathrm{i}=8}\left|\mathrm{E}_{\mathrm{i}}\right|}{\sum_{\mathrm{i}=5}^{\mathrm{i}=8}\left|\mathrm{E}_{\mathrm{i}}\right|}=\frac{528-504}{528}=1 / 22
$$

and therefore ordinary is given by:

$\mathrm{E}(\mathrm{O})=\left(\gamma_{\mathrm{o}}\right) \mathrm{mc}^{2}=\mathrm{mc}^{2} / 22$

exactly as expected from previous analysis. Dark energy on the other hand is squarely connected to the negative energy of the non-vanishing torsional part in the Cartan connection, namely the 504 known also from the particle physics spectroscopy of Heterotic string theory. The corresponding Lorentzian factor is thus:

$\gamma_{\mathrm{D}}=-(504) /(528)=-\sum_{\mathrm{i}=5}^{\mathrm{i}=8}\left|\mathrm{E}_{\mathrm{i}}\right| / \sum_{\mathrm{i}=4}^{\mathrm{i}=8}\left|\mathrm{E}_{\mathrm{i}}\right|$

leading to a dark energy density:

$\mathrm{E}(\mathrm{D})=-(504) / 528) \mathrm{mc}^{2}=-\mathrm{mc}^{2}(21 / 22)$.

This is exactly the same result which we find when using 'talHooft's dimensional regularization $\mathrm{D}-4=\in$ when setting $\in=\mathrm{k}=2 \phi^{2}$ and finding an entangled energy density:

$\mathrm{E}(\mathrm{D})=\left(\frac{4-\mathrm{k}}{4}\right) \simeq \mathrm{mc}^{2}(21 / 22)$.

Einstein's energy density $\mathrm{E}=\mathrm{mc}^{2}$ on the other hand is blind to the preceding fine distinction which wrongly considers $4 / 4=1$ completely equivalent to $528 / 528=1$, and is therefore given by the sum of the absolute value of both energies as :

$\mathrm{E}($ Einstein $)=\mathrm{E}(\mathrm{O})+|\mathrm{E}(\mathrm{D})|$

$=\mathrm{mc}^{2}\left(\frac{1}{22}+\frac{21}{22}\right)=\mathrm{mc}^{2}$.

In other words Einstein's maximal energy formula does not need to be quantumally corrected but only quantumally dissected into two parts.

Now we could make another profound interpretation of this result if we consider $\mathrm{E}$ (Einstein) to be unity by setting $\mathrm{m}=\mathrm{c}=1$. That way the dark energy could be viewed as a
Legendre transformation of ordinary energy, that is to say it is a complimentary energy as far as the absolute value is concerned. In other words, dark energy is the negative value of the complimentary energy or the ordinary measurable energy. We note on passing that 528 is divided in Witten's model into 1D strings, 2D membranes and 5D Branes [15, 84, 105]:

$\mathrm{N}_{\mathrm{K}}^{(32)}=\left(\begin{array}{c}11 \\ 1\end{array}\right)+\left(\begin{array}{c}11 \\ 2\end{array}\right)+\left(\begin{array}{c}11 \\ 5\end{array}\right)=11+55+462=528$

The corresponding E8E8 expression includes the pointlike particles as well as the 3D and 4D Branes:

$\mathrm{N}(\mathrm{E} 8 \mathrm{E})=\left(\begin{array}{c}11 \\ 0\end{array}\right)+\left(\begin{array}{c}11 \\ 3\end{array}\right)+\left(\begin{array}{c}11 \\ 4\end{array}\right)=1+165+330=496$

$=|\mathrm{E} 8 \mathrm{E} 8|$.

The hidden Yano-killing 504 on the other hand are given in Heterotic super string theory by three groups of states, namely 480, 16 and 8 leading to:

$480+16=496$

and

$496+8=504$

as explained in great detail in $[28,36]$. We also note that $\mathrm{N}_{\mathrm{K}}{ }^{(32)}+\mathrm{N}(\mathrm{E} 8 \mathrm{E} 8)=1024$ while $\mathrm{d}_{\mathrm{c}}{ }^{(11)}=1024$ for $\mathrm{d}_{\mathrm{c}}{ }^{(0)}=(1 / 2)$ and $\sum_{\mathrm{i}=0}^{\mathrm{i}=11}\left(\begin{array}{c}11 \\ \mathrm{i}\end{array}\right)=(2)(1024)=2048$. Note also that in various Heterotic string theories different divisions exist. For an in depth study of the E-line exceptional Lie symmetry groups at the root of the present theory Refs. [30-32] could be considerably helpful.

\section{ELEMENTARY DERIVATION OF EINSTEIN'S REVISED FORMULA FOR ORDINARY ENERGY E = $\mathrm{MC}^{2} / 22$}

Evidently when Einstein drove his famous $E=\mathrm{mc}^{2}$ he did not write a Lagrangian [24]. However supposed he knew how to do what is according to current prejudice the only acceptable way forward, namely writing down a Lagrangian [99]. First such a Lagrangian would lead in our opinion to a few realizations. For a start the only degree of freedom from a particle physics viewpoint would be the only messenger particle known at the time of Einstein, namely the photon. Energy on the other hand would be the Eigenvalue of a Schrödinger equation however not that of a particle but rather that of the entire universe [24]. Thus E of Einstein would be the Eigenvalue of an unknown quantum gravity Schrödinger-like equation. However we know in the meantime that the physics of our universe is best approximated by at least 12 photon-like particles and not only one photon. On the other hand we know very well that reducing a 12 degrees of freedom Lagrangian to only one degree of freedom Lagrangian would lead to a gross over estimation of the corresponding Eigenvalue, i.e. the energy E of Einstein which is a 
well known theorem by Lord Rayleigh [25]. Consequently we see that $\mathrm{E}=\mathrm{mc}^{2}$ is much larger than what a locally 12 degrees of freedom Lagrangian allows. So much for the qualitative situation. The quantitative one is more involved. Never the less an educated guess leads to the following line of reasoning. Noting that the kinetic energy of Newton $E_{N}=$ $\frac{1}{2} m v^{2}$ and $\mathrm{E}$ of Einstein differs mainly by a "scaling" factor $(1 / 2)$ when disregarding the limit $\mathrm{v} \rightarrow \mathrm{c}$ and noting also that self similarity is a fundamental aspect of both the macro (solar system) and the micro cosmos (Bohr atom) then one is encouraged to think that $\mathrm{E}=\mathrm{mc}^{2}$ could be scaled down proportionately to $\mathrm{E}=\mathrm{mc}^{2} / 22$, where $1 / 22$ is the scaling factor [27]. The value 22 could be thought of in two different obvious ways. It is the 26 bosonic dimensions of the Veneziano spacetime minus Einstein's 4 dimensional spacetime, i.e.:

$\lambda=1 /(26-4)=22$,

or alternatively we use $\mathrm{E}_{\mathrm{N}}=\frac{1}{2} \mathrm{mv}^{2}$ and invoke the scaling:

$\lambda=1 /[|\mathrm{SU}(3) \mathrm{SU}(2) \mathrm{U}(1)|-\gamma]$

$=1 /(12-1)=1 / 11$

and the limit $\mathrm{v} \rightarrow \mathrm{c}$ to find that [87-89]:

$\left.\mathrm{E}_{\mathrm{N}} \rightarrow \mathrm{E}(\mathrm{O})=\lambda\left(\frac{1}{2} \mathrm{~m}(\mathrm{v} \rightarrow \mathrm{c})^{2}\right)\right)$

$=\left(\frac{1}{11}\right)\left(\frac{1}{2}\right) \mathrm{mc}^{2}=\frac{1}{22} \mathrm{mc}^{2}$.

The preceding plausibility derivation could be made mathematically water tight in various ways discussed in previous publications and will not be followed here any further in order not to lose the main thread of the present work and its objective.

5. DETAILS OF RINDLER SPACE CALCULATIONS LEADING TO THE AREAS $\phi^{5} / 2$ AND $5 \phi^{2} / 2$ OF ORDINARY AND DARK ENERGY RESPECTIVELY

We follow Fig. (2) which represents a Rindler space and the associated horizon [17, 29, 30]. In the following analysis we concentrate on the questions pertaining to measure theory, i.e. the various Lorentzian invariant hyperbolic areas and for the moment relegate the question of physical interpretation to a back seat. We see that we have three distinct areas. The first $\mathrm{A}_{\mathrm{o}}$ is the total area of the large triangle, $\mathrm{H}, \mathrm{P}, \mathrm{P}^{1}$. Calculating the area of $\mathrm{A}_{o}$ is truly trivial since it consists of two symmetric triangles leading to $[9,17]$ :

$A_{o}=2\left[\frac{1}{2} t(\eta / 2) Z(\eta / 2)\right]$

where $\eta$ is the opening angle of the Rindler wedge [17,29,30]. Since: $t(\eta)=1 \sinh (\eta)$

and

$Z(\eta)=1 \cosh (\eta)$

We can write $A_{o}$ as:

$A_{o}=l^{2} \cosh (\eta / 2) \sinh (\eta / 2)$

where $l=1 / \mathrm{a}$ is the distance between the Rindler horizon and the observer as seen by him and a is the constant Rindler acceleration and a is the Rindler constant acceleration [29, 30].

The second area is the symmetric hyperbolic segment $A_{1}$ as shown in Fig. (2) [20, 30]. This gives twice the integral of half of the segment as:

$A_{1}=2 \int_{0}^{\eta / 2} \sinh \left[l^{2} \sinh (\eta / 2) \cosh (\eta / 2)-\eta / 2 l^{2}\right]=$

$5 \phi^{2} / 2$

Since:

$Z(\eta)=1 \cosh (\eta)$

then:

$\frac{d z \quad(\eta)}{d \eta}=1 \operatorname{subg}(\eta)$

and therefore:

$d Z(\eta)=l \sinh (\eta) d \eta$.

Inserting one finds:

$A_{1}=2 \int_{0}^{\eta / 2} t(\eta) d z \quad(\eta)=2 \int_{0}^{\eta / 2} \ell \sinh (\eta) l \sinh (\eta) d \eta$

$=2^{2} l \int_{0}^{\eta / 2} \sinh (\eta)^{2} \mathrm{~d} \eta$.

This is a straighforward simple integration but could also be found in any standard handbook of mathematics to be:

$$
\begin{aligned}
& A_{1}=2 l^{2}\left[\frac{1}{2} \sinh \eta \cosh \eta-\frac{1}{2} \eta\right]_{0}^{\eta / 2} \\
& =l^{2}[\sinh \eta \cosh \eta-\eta]_{0}^{\eta / 2} \\
& =l^{2}(\sinh \eta / 2)(\cosh \eta / 2)-(\eta / 2)\left(l^{2}\right) .
\end{aligned}
$$

Finally following Fig. (2) the area of the hyperbolic triangle A2 is found simply as the difference between $A_{o}$ and $\mathrm{A}_{1}$. Consequently:

$A_{2}=A_{o}-A_{1}=l^{2} \cosh (\eta / 2) \sinh (\eta / 2)-$

$\left[\ell^{2} \sinh (\eta / 2) \cosh (\eta / 2)-\eta / 2 \ell^{2}\right]=\left(l^{2} \eta\right) / 2$ 


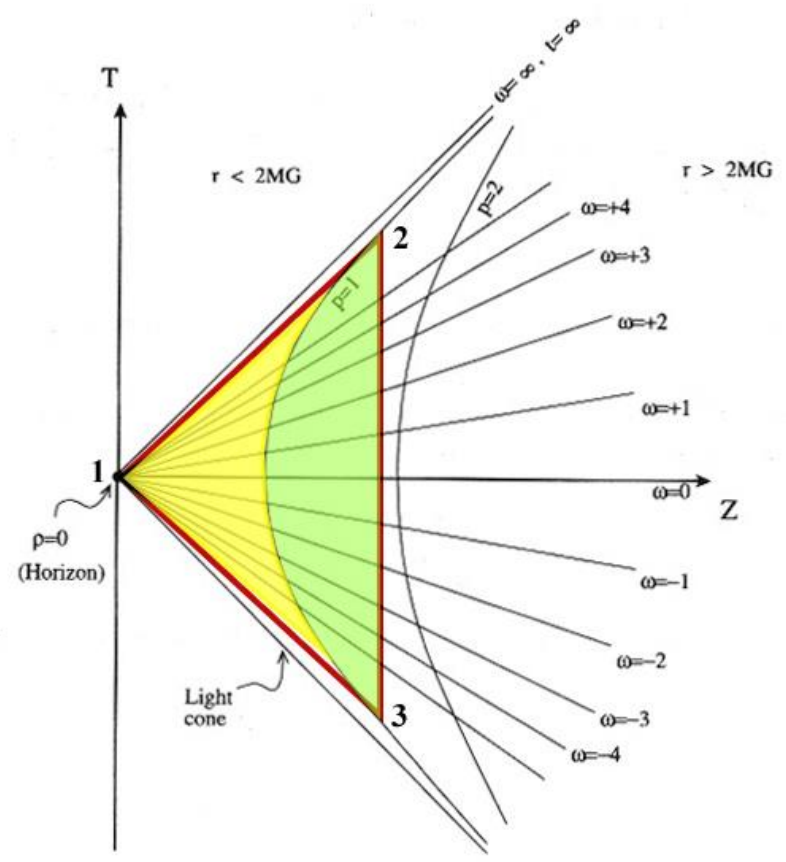

Fig. (2). Equal time and proper distance surfaces in Rindler space $[9,17,29,30]$.

It is one of the fundamental results of the unit interval "topological" physics introduced in earlier work that $\mathrm{c}=\phi$ and $m=\phi^{3}$. On the other hand a few moments of deep reflection will reveal that $\mathrm{m}=\eta$ and $\mathrm{c}=\ell$. Consequently:

$\eta=\phi^{3}$ and $\ell=\phi$.

Inserting in $\mathrm{A}_{2}$ one finds:

$\mathrm{A}_{2}=\phi^{5} / 2$.

That immediately leads to our second most important result, namely:

$\mathrm{A}_{1}=5 \phi^{2} / 2$

where $\phi=(\sqrt{5}-1) / 2$. It is a trivial matter to see that rounding the value of $A_{1}$ and $A_{2}$ to the nearest integer gives us the "exact integer" value of the density factor of ordinary energy $\mathrm{A}_{2} \simeq(1 / 22)=\gamma_{2}$ and dark energy $\mathrm{A}_{2} \simeq(21 / 22)=\gamma_{1}$. This is the same result of preceding sections.

\section{PINCHING SPACETIME}

Various experiments with pinched elastic and elastoplastic cylindrical shells were actually performed long ago (see Fig. 1) [74]. In fact it is extremely easy to demonstrate the effects of induced local change of curvature causing a considerable distance away a change of curvature of opposite sign [15]. For that we need nothing more than a large sheet of writing paper rolled into a long cylinder and squeeze it in the middle as described in previous work. That way we establish at a minimum an analogy connecting not only engineering metal forming with cosmology but also with thermodynamics. The analogy makes it plausible that local attractive deformation causes anti-attraction far away from the local opposite sign attraction. Curiously the Master Thesis of the Author was about physical nonlinearity of torsion in some elastic structures [114]. That is exactly what is missing in Einstein's geometry and that is exactly what Cosserat and Cartan provide. Also by coincidence or providence the Ph.D. of the Author forty years ago was on the effect of imperfection sensitivity on unstable points of bifurcation of elastic shells which is a classical counterpart to quantum wave collapse and missing dark energy [113].

\section{SELF SIMILARITY, P-ADIC QUANTUM PHYSICS AND CANTORIAN SPACETIME}

Integers are possibly the simplest source of self similarity in physics. A trillion is nothing but unity scaled up a trillion times. Number theory is of course very close to the continuum hypothesis and consequently the most fundamental question regarding the nature of space and time. It is therefore important to understand the intimate relation of the present paper with the fundamental result found by the school of PAdic quantum physics which we discussed in some details on previous occasions $[37,40]$. We stress that only zero and infinity are not ordinary numbers but deep mathematicalphilosophical concept. Since unity differs only by a scaling factor we see the fundamental meaning of the unit interval physics $[79,88]$ where the speed of light is a natural topological quantity $\mathrm{c}=\phi$. 


\section{DARK ENERGY AND DARK MATTER SEGRE- GATED AND UNIFIED}

On the most fundamental level of transfinite set theory we have only the ordinary energy connected to the quantum zero set particle, i.e. $\mathrm{E}(\mathrm{O})=\left(\phi^{5} / 2\right) \mathrm{mc}^{2}$ which is directly proportional to the area of the hyperbolic triangle of the Rindler wedge $\mathrm{A}_{2}$ and the dark energy connected to the quantum empty set of the wave proportional to the circular segment area $A_{1}=1-A_{2}=5 \phi^{2} / 2$. Clearly not all of $A_{1}$ dark energy is pure energy but some of it is dark matter exactly as part of the ordinary energy is ordinary matter expressed in terms of energy following the theoretical insight of Einstein and the essence of his formula if not its exact quantitative prediction which needed the present revision. We are not yet in a position to give a stringent mathematical distinction between dark energy and dark matter, which although lumped together in the energy of the five dimensional empty set theory, has different physical effects and manifestation. However what we can do here is to give a logically coherent plausibility explanation converging towards a mathematical watertight explanation for the difference between dark energy and dark matter [27].

Let us recall first that our previous calculations demonstrated that while $4.5 \%$ of the energy density of the cosmos is measurable ordinary energy and matter, the rest, i.e. 100 $-4.5=95.5 \%$ of the energy density must be in the form of dark energy which we eblieve to be responsible for the initially surprising astrophysical observations connected to the accelerating cosmic expansion in addition to dark matter which we presume to be responsible for various astronomical anamolous observations. Let us further recall that our fundamental equation from which we construct our most fundamental coupling constant, namely $\bar{\alpha}_{o} \cong 137$ is found from $[17,21,27]$;

$$
\begin{aligned}
& \bar{\alpha}_{o}=\bar{\alpha}_{1}(1 / \phi)+\left(\bar{\alpha}_{2}=\bar{\alpha}_{1} / 2\right)+\bar{\alpha}_{3}+\bar{\alpha}_{4}=(60)(1 / \phi)+ \\
& 30+9+1 \\
& =137+\mathrm{k}_{\mathrm{o}}=137+\phi^{5}\left(1-\phi^{5}\right)=137.0820393
\end{aligned}
$$

where $\phi=(\sqrt{5}-1) / 2$ and $\bar{\alpha}_{4}=\bar{\alpha}_{\mathrm{QG}}=1$ is the largest possible quantum gravity inverse coupling. The next step in our plausibility "derivation" is to notice that $\bar{\alpha}_{1}+\bar{\alpha}_{2}+\bar{\alpha}_{3}$ $+\bar{\alpha}_{4}=100$ and that this sum could be viewed as a normed value for summing over all the infinite dimensions spanning the fractal-Cantorian spacetime of our theory. In other words this 100 is a normed value for the number of internal as well as external dimensions or brocken symmetries. Now we divide these dimension into three categories. First the "visible" dimenhsion, i.e. the 3 space dimension plus the time dimension of our classical daily experience. The second cetegory of dimensions are the compactified 22 left from the bosonic Nambu-Veneziano strong interaction dimension. The third category of dimensions are the diluting rest, i.e. $100-(22+4)=100-26=74$ which represents a finite value for the infinitely many fractal dimensions spanning our fractal spacetime. The next step in our explanation is now quite obvious. We hold it that the various percentages of the energy density of the universe are based on the preceding categorical subdivision of the various normed expectation numbers of the spacetime and internal dimensions. In other words, the four dimensions of spacetime correspond to 3 percent ordinary matter and 1 percent ordinary energy and radiation making up $4 \%$ altogether. The 22 compactified dimensions on the other hand correspond to a $22 \%$ dark, i.e. "compactified" matter. Finally we are left with the well hidden and diluted rest, namely $100-(4+22)=74 \%$ truly pure dark energy responsible for the negative pressure behind the observed accelerated cosmic expansion. Neddless to say these results, taken on face value, are simple integer approximations of the various cosmological measurements which the majority of put ordinary energy at $4.5 \% \simeq 4 \%$, dark matter $22 \%$ and dark energy $74.5 \simeq 74 \%$ [32, 47-51].

The interesting question on the fundamental level of set theory is to ask how the empty set splits into two sets separating pure dark matter from pure dark energy. Our guess is that it is a very similar phenomenon and analysis to that leading to phase transition from purely ordinary matter to purely ordinary energy [24]. The set theoretical analysis behind the preceding illucidation is currently in progress but we decided to release the present incomplete information in the hope of attracting more thinking in this direction.

\section{TOPOLOGICAL DEFECTS, TEXTURE AND THE EMPTY SET}

An extremely powerful mathematical subject which benefited cosmology is understanably algebraic topology. Without going into any detail we note the information given in the Table $\mathbf{1}$ and add the conjecture that the dimension of texture is -1 and that it could be extrapolated to mean an empty set-like Cantorian wild topology akin to Alexander Horns. Texture in this interpretation corresponds to dark energy and the negative sign to a negative cosmological constant [52].

\section{THE FUNDAMENTAL ROLE OF HARDY'S QUANTUM ENTANGLEMENT}

The fundamental importance of the theoretical discovery of Hardy's probability of quantum entanglement $\mathrm{P}($ Hardy $)=$ $\phi^{5}$ and its subsequent accurate experimental verification cannot be stressed enough $[7-9,19,22,70,71]$. At a minimum the present work and the understanding of the essence and meaning of dark energy could not be understood in its full ramifications without the quantum entanglement of the cosmos. Without repeating previous arguments and analysis, we just recall for the sake of completeness that $\mathrm{E}(\mathrm{O})$ and E(D) could be interpreted and written in terms of Hardy's quantum entanglement as:

$\mathrm{E}(\mathrm{O})=\mathrm{P}($ Hardy $)\left(\frac{1}{2} \mathrm{~m}(\mathrm{v} \rightarrow \mathrm{c})^{2}\right)=\left(\phi^{5} / 2\right) \mathrm{mc}^{2}$ 
and

$\mathrm{E}(\mathrm{D})=1-\left(\mathrm{E}(\mathrm{O})=\left(5 \phi^{2} / 2\right)\left(\mathrm{mc}^{2}\right)\right.$.

\section{INTERMEDIATE DISCUSSION}

In a sense we are dealing here with a cosine of the butterfly effect of E. Lorentz and O. Rössler. A small feeble local effect in the form of an attractive gravity induces at infinity an accumulated effect of anti-gravity adjacent to the horizon [149]. In a sense exotic ideas that the universe may resemble a giant black hole or a knot compliment at infinity and therefore neither open nor closed but topologically clopen may not be that far off after all. That way cosmological data and observation collected over a very long period culminating in several deserving Nobel Prizes in Physics has fused various theories together and confirmed the reality of Hawking's radiation, Unruh's temperature, anti-gravity and Rindler spacetime all apart of completing the magnificent work of Einstein's relativity, Planck-Bohr-Heisenberg's quantum mechanics and Boltzmann thermodynamics as indicated in the eminent work of T. Padmanabhan and his school [32, 33]. One could of course argue that the part of the present derivation which is based on an analogy between metal forming, pinching of elastic tapes and the real behavior of a material spacetime micropolar elasticity is less fundamental than previous derivations starting from the zero set as a prequantum particle and the empty set as a pre-quantum wave [36-38]. However this is a largely subjective judgment and a matter of taste and personal philosophical stance. In fact one could view the difference between the negative dimension of the empty set $\mathrm{D}_{\mathrm{T}}=-1$ as well as the $\mathrm{D}=-1$ degree of freedom of pure gravity for $\mathrm{d}=2$ and the cosmological constant $\Lambda=-1$ as mere mathematical and physical tautology. We have to admit that because of space limitation we have hardly touched upon many other vital points that could have enhanced understanding the magnificent interconnectivity of mathematics, high energy physics and cosmology leading to the present synthesis. For instance we did not discuss the role of symplectic geometry [66] that would have made the appearance of the golden mean and its derivatives and powers everywhere in our theory plausible, even unavoidable. However the reader may find all these points and more adequately covered in Refs. [63-70].

\section{CAPILLARY SURFACE ENERGY ELUCIDATION OF THE COSMIC DARK ENERGY - ORDINARY ENERGY DUALITY}

This short section reports on an unsuspected and quite surprising connection between capillary forces and dark energy. As the reader realized from the previous sections and as is evident from numerous previous publications a fundamental theory was advanced to explain the baffling cosmic observation associated with conjectured dark energy and the surprising measured accelerated rather than decelerating expansion of the universe. Our most rigorous theory was an exact calculation based on particle-wave duality in highly mathematical set theoretical formulation led to an ordinary measurable energy density of $\mathrm{E}(\mathrm{O})=\mathrm{mc}^{2} / 22$ where $\mathrm{m}$ is the mass and $c$ is the speed of light, i.e. only 1/22 of Einstein's famous energy density [4]. This was a remarkable result and in full agreement with the latest and most accurate cosmic measurements and supernova analysis which led to the award of several Nobel Prizes in Physics on two different occasions. For dark energy the density found and reconsidered here was $E(D)=m c^{2}(21 / 22)$ which amounts to exactly $1-\mathrm{E}(\mathrm{O})$ showing with absolute clarity that Einstein's density, lacking the quantum component, is blind to any distinction between ordinary energy and dark energy. Thus apart of the quantitative resolution of this major problem, a fundamental conclusion was reached elevating Einstein's relativity formula $\mathrm{E}=\mathrm{mc}^{2}$ to a quantum relativity equation $\mathrm{E}=$ $\left(m c^{2} / 22\right)+m c^{2}(21 / 22)=m c^{2}$ where $E(O)$ is the ordinary energy of a quantum pre-particle in a five dimensional Kaluza-Klein spacetime and E(D) is the negative dark energy of the quantum pre-wave in the same Kaluza-Klein spacetime [34]. Seen in this way we begin to understand why ordinary positive energy could be detected and measured while the negative dark energy could not, at least not directly or using any conventional method. The reason for this failure is as simple as it is unexpected and is anchored in the deep logic of set theory. A quantum particle is in set theoretical terms a physical materialization of the zero set. The quantum wave on the other hand is the physical materialization of the empty set $[35,36]$. Since "measurement" interferes with the empty set and causes it to become non-empty, the empty quantum "wave" set transmutes instantly to a zero quantum "particle" set at measurement. This is what we call wave collapse and that is why the negative dark energy of the wave cannot be measured in the ordinary way unless wave non-demolition measurements could be developed in the future [86].

The preceding set theoretical explanation, although mathematically and logically accessible and in some sense even intuitive, cannot be called physically obvious. For instance it is true that we have a clear picture of a particle with a wave as its cobordism, i.e. as its surface. Never the less, particles and surface, although inseparable, cannot be dealt with experimentally except via the contra-intuitive perspective of wave-particle duality. All the same it would be more than desirable to have a conjugate more down to earth and conventional physical picture to go hand in hand with the fundamental set theoretical interpretation just outlined.

In the present work we think that we have at long last found a parallel physical interpretation to our set theoretical picture that is in a one to one correspondence with the zero set-empty set particle-wave duality. This we explain next.

Let us consider a capillary surface [82] which is something well known in fluid mechanics and in fact from various simple experiments which almost everyone encountered in elementary school physics. On a fundamental level however the phenomena involves very complex nonlinearity effects and is related to the theory of a minimal surface. The point is that the energy on the surface is meta-stable and is susceptible to spontaneous symmetry breaking bifurcation instability by jumping into a much lower energy state similar in principle to phase transition as well as local buckling of thin 
walled structures, a field in which the present author was initially trained and specialized. As we said earlier the subject is also closely related to minimal surfaces [83] and we note an almost esoteric property of capillary surfaces which is that although real, they have no thickness at all. This is somehow an unexpected bridge between the pure mathematics of transfinite set theory and the real physics of capillary fluid mechanics. We note further that despite the fact of being meta-stable, capillary surfaces are remarkably persistent in some experiments [82] which makes a good analogy to the steady state propagation of a quantum wave.

To sum up we could look upon dark energy, which is the negative energy of the quantum wave surface of the quantum particle core, as being analogous to the physically and classically real capillary surface energy which cannot always be easily measured due to spontaneous jump into the lower energy level of the core. Here we are speaking of higher and lower in absolute terms and are of course disregarding the sign convention. We conclude by noting the immense importance of relativistic hydro-dynamical models in physics and astrophysics [106].

\section{DARK ENERGY FROM PURE GRAVITY}

In the present section which maybe the most important of the entire paper, we start from the basic concept of pure gravity, i.e. gravity in the total absence of any matter field [107]. The well established relevant equation in this case connecting the field theoretical degrees of freedom $\mathrm{D}$ of pure gravity to the dimension of the space $d$ is given by [108, 109]:

$\mathrm{D}=\mathrm{d}(\mathrm{d}-3) / 2$.

Thus for the fundamental situation of $d=2$ corresponding in string theory for instance to the string world sheet [117] we have the remarkable negative value $\mathrm{D}=-1$ for what we called degrees of freedom [69]. This is formally identical to the Menger-Urysohn topological dimension of the empty set $[12,27]$ :

$\mathrm{D}($ empty $)=\left(\mathrm{D}_{\mathrm{T}}, \mathrm{D}_{\mathrm{H}}\right)=\left(-1, \phi^{2}\right)$

where $\mathrm{D}_{\mathrm{H}}$ is the Hausdorff component and $\phi=(\sqrt{5}+1)$ and conceptually this negative degree of freedom has almost the same essential meaning of an empty set. We should stress again that a negative degree of freedom makes no physical sense at all and little if any mathematical sense that is unless it is understood as an empty set. Further more for the classical case of $d=3$ we have obviously a practically zero set $\mathrm{D}=$ 0 corresponding to $[20,87]$ :

$\mathrm{D}($ zero $)=\left(\mathrm{D}_{\mathrm{T}}, \mathrm{D}_{\mathrm{H}}\right)=(0, \phi)$.

Lifting the Hausdorff dimension component of both the empty "pure" gravity and the "zero" gravity to five dimensional Kaluza-Klein spacetime, we find the following pseudo volume, namely [20, 87]:

$\operatorname{Vol}_{(5)}($ pure gravity $)=\phi^{2}+\phi^{2}+\phi^{2}+\phi^{2}+\phi^{2}=5 \phi^{2}$

and
$\operatorname{Vol}_{(5)}($ zero gravity $)=(\phi)(\phi)(\phi)(\phi)(\phi)=\phi^{5}$

$=\mathrm{P}($ Hardy $)$.

Noting the volume interpretation of the Hausdorff dimension our total volume, i.e. that modeling the 5D quantum wave of pure gravity and that modeling the $5 \mathrm{D}$ quantum particle of zero gravity, one finds [20, 21, 87]:

Total $\operatorname{Vol}_{(5)}=5 \phi^{2}+\phi^{5}=2=\operatorname{dim}$ (string world sheet).

The relative density for pure gravity corresponding to the wave is therefore:

$\gamma_{\mathrm{w}}=5 \phi^{2} / 2$

while that corresponding to the particle is clearly:

$\gamma_{\mathrm{p}}=\phi^{5} / 2$

Inserting in Einstein's energy density we find both the ordinary measurable energy:

$\mathrm{E}(\mathrm{O})=\gamma_{\mathrm{p}} \mathrm{mc}^{2}=\left(\phi^{5} / 2\right) \mathrm{mc}^{2} \simeq \mathrm{mc}^{2} / 22$

and the "meta" dark energy:

$$
\begin{aligned}
& \mathrm{E}(\mathrm{D})=\gamma_{\mathrm{w}} \mathrm{mc}^{2} \\
& =\left(5 \phi^{2} / 2\right) m c^{2} \\
& =m c^{2}(21 / 22)
\end{aligned}
$$

Exactly as expected [34-36]. Thus the fact that empty Einstein space is so rich on structures and the equality of the degrees of freedom of massless graviton and that of pure gravity indicate that Einstein's relativity is closer to quantum mechanics than we ever imagined [23, 24, 100]. It is an extremely satisfying insight into the blue print of nature to observe that the Newtonian kinetic energy formula can be defined within our present theory as the average of fusing ordinary energy and dark energy at low velocities.

\section{THE EFFECT OF GRAVITY ON RUNNING THE COUPLING CONSTANT AND 'THOOFT'S DIMEN- SIONAL REGULARIZATION $[119,120]$}

Ignoring the effects of gravity at grand unification energy scale is questionable. By contrast ignoring the effects of gravity in the running of coupling constants of gauge forces near the Planck scale is totally wrong [15, 21-24]. By Tduality the same is true at the opposite extreme, namely cosmic scales $[6-8,21,22]$. These facts are well known and understood in E-infinity Cantorian spacetime theory and without going into detail, we just mention a few important facts due to their importance for the analysis in this section. First the theoretical E-infinity electromagnetic fine structure constant could be reconstructed correctly only when $\bar{\alpha}_{\mathrm{Q}}=1$ of quantum gravity is included [21, 27, 37]:

$\bar{\alpha}_{\mathrm{o}}=\left(\bar{\alpha}_{1}\right)(1 / \phi)+\left(\bar{\alpha}_{2}=\bar{\alpha}_{1} / 2\right)+\bar{\alpha}_{3}+\left(\bar{\alpha}_{4}=\bar{\alpha}_{\mathrm{Q}}\right)=$ $(60)(1 / \phi)+30+9+1=137+\mathrm{k}_{\mathrm{o}}=137.082039325$

$\simeq 137$. 
Second the Heterotic string dimensional hierarchy starts with $\left(\bar{\alpha}_{\mathrm{o}} / 2\right)$ multiplied with $\phi^{\mathrm{n}}$ to generate after 8 steps the following values $[120,121] 42+\mathrm{k}, 26+\mathrm{k}, 16+\mathrm{k}, 10,6+\mathrm{k}$, $4-\mathrm{k}$. Clearly $4-\mathrm{k}$ where $\mathrm{k}=\phi^{3}\left(1-\phi^{3}\right)=0.18033989$ is the fractal Hausdorff dimension at the corresponding Planck and Hubble scale.

\section{ON SOME POSSIBLE QUASI-ELEMENTARY PARTICLES MEDIATING QUANTUM ENTANGLE- MENT AND DIMENSIONAL REGULARIZATION}

\subsection{Dimensional Regularization Revisited}

'tHooft-Veltman-Wilson dimensional regularization is a highly successful method in its analytical form as well as in the computerized counterpart extensively used in solid state physics [119-130]. It is generally known that the three Nobel Laureates mentioned above occasionally asked themselves if the use of $D=4-\varepsilon$ for spacetime where much smaller than unity could imply that spacetime may be a fractal at the quantum scale [123-134]. In fact the present author knows firsthand that G. 'tHooft and at the time his thesis Adviser, M. Veltman debated this issue but did not come to any conclusive result [123-127]. Historians of science may well debate the issue of why this relatively small step from $\mathrm{D}=4-\varepsilon$ to a fractal spacetime described by a Hausdorff dimension was not discovered much earlier i.e. at the same time when the renormalizability of the Yang-Mills theory was discovered [120, 128]. A possible explanation and we stress that it is only a possibility, may be that the intimate link between Borel summability and fractals was not obvious. However the fact is that Emil Borel in France [136] was quite an expert on the essence of fractals long before it was popularized and named fractals by B. Mandelbrot. It is a firm historical fact that G. Cantor's ideas and his transfinite set theory survived the onslaught of the mainstream at the time and moved from there to France and finally found a dedicated group which established the famous Moscow School of Mathematics [136] led by Egerov. Many of the towering figures of modern mathematics were members of this school and we may mention here the names of a few who had considerable influence on the development of mathematics and theoretical physics in general and E-infinity Cantorian spacetime in particular, namely Kolmogorov, Suslin and Urysohn (see E-infinity communications [135-140]). Equipped with the mathematical machinery of E-infinity theory and having some knowledge of quantum field theory and 'tHooft's method it was relatively easy for the present author to discover the almost one to one correspondence of dimensional regularization and the Cantorian-fractal theory of high energy physics [138-153]. We stress once more that the subtlety of dimensional regularization lies in the application of Borel's method for treating divergence and that is where Cantor sets and KAM theorem [137] enters into the subject leading to the inescapable conclusion that 'tHooftVeltman-Wilson method implies a Cantorian fractal spacetime and what more exciting also to a host of exotic new elementary particles [142-144] as we hope to show in the following main part of the ensuing sections of the present short paper.

\subsection{Dimensional Regularization and Dark Energy}

To avoid troublesome singularities and to be able to extract a finite answer from an otherwise diverging series, dimensional regularization resorts to some ingenious mathematics due to E. Borel [123, 124, 144] Not only that but the mathematical scheme would also require that one thinks of the four dimensionality of our spacetime as being slightly less than 4 , namely $4-\varepsilon$ where $\varepsilon=1$ will play the role of an order parameter, i.e. more or less a perturbation parameter [119-132]. In E-Infinity we encountered in the context of a fractal Kaluza-Klein [145] the fractal K-K spacetime dimension given by [146]:

$\mathrm{D}_{\mathrm{F}}=5+\phi^{3}$

This could be considered to approach $\mathrm{D}=5$ from above with $\phi^{3}$ being an order parameter with fixed value where $\phi=2 /(1+\sqrt{5})$ as discussed in great detail in [144-146]. The sparseness of $5+\phi^{3}$ compared to 5 was subsequently reasoned geometrically to be the density of the dark energy of our cosmos provided the $\mathrm{K}-\mathrm{K}$ fractal theory is an accurate topological description of our universe. In this respect our hope was greatly fulfilled and we were rewarded by a result in full agreement with cosmic measurements and observations as well as all previous derivations, namely [147, 148]:

$\gamma(\mathrm{D})=5 /\left(5+\phi^{3}\right) \simeq 95 \%$.

As mentioned a moment ago the situation with $\mathrm{D}=4-\varepsilon$ is quite similar and we set $\varepsilon$ to be naturally related to Hardy's entanglement for a single particle of two entangled Hardy quantum particles, i.e. $\mathrm{P}($ Hardy $) / 2=\phi^{5} / 2$ then multiply this value with each of the four dimensions of our spacetime and that way we find $\varepsilon$ namely [4, 5, 27-29]:

$$
\begin{aligned}
{[\mathrm{P}(\mathrm{H}) / 2] } & (4)=2 \phi^{5} \\
= & \varepsilon \\
= & \mathrm{k}
\end{aligned}
$$

where $\mathrm{k}=\phi^{3}\left(1-\phi^{3}\right)=0.18033989$. The dark energy density could now be determined as in the $\mathrm{K}-\mathrm{K}$ theory to be the ratio of the 'regulated' dimension $\mathrm{D}=4-(\varepsilon=\mathrm{k})$ to the original dimension, namely 4 . That means $[121,123]$ :

$$
\begin{aligned}
\gamma(D) & =\frac{4-(\varepsilon=\mathrm{k})}{4} \\
& =\frac{4-(0.18033989)}{4} \\
& =1-\left(\phi^{5} / 2\right) \\
& =95.5 \%
\end{aligned}
$$

which is exactly our previous result apart from being what was measured by WMAP and Planck. 
The rational question is now to ask what kind of magical number system is involved in the preceding calculation and how come that this numeric fits seamlessly to physics and everything else? This we explain in the next section and we hasten to say that at the end we should find out that the magic is nothing else but the revival of Kantian pure mathematical reasons upon which our very existence is based.

\subsection{Replacing Borel Summation [144] by Suslin Oper- ation and Weyl Scaling [44]}

Let us reconsider our last result for $\gamma(\mathrm{D})$ which after some simple manipulation could be written as:

$$
\begin{aligned}
\gamma(\mathrm{D}) & =1-\left(\phi^{5} / 2\right) \\
& =5 \phi^{2} / 2 \\
& =\frac{21+\mathrm{k}}{22+\mathrm{k}}
\end{aligned}
$$

Noting that we started with:

$\mathrm{D}=4-\mathrm{k}$

we see that we can let $\mathrm{k} \rightarrow 0$ without losing the integer part of our result i.e. equation 5 and 6 . Thus for $\mathrm{k} \rightarrow 0$ we have:

$\mathrm{D}=4-\mathrm{k} \rightarrow 4$

and

$\gamma(\mathrm{D})=\frac{21+\mathrm{k}}{22+\mathrm{k}} \rightarrow \frac{21}{22}$

However if we had worked from the very beginning with $\mathrm{D}=4-0$ we would have obtained the trivially wrong result, namely:

$$
\begin{aligned}
\gamma(\mathrm{D}) & =\frac{4}{4} \\
& =1
\end{aligned}
$$

Again, how is this possible? The short answer is that we used one of the seven pillars of wisdom which is traditionally ignored in physics, namely the number system employed by nature to construct a logical universe rather than the various mundane number systems which are based on the human experience in dealing with everyday life and that includes the rather rudimentary binary system of digital computers namely of zero and one [18, 22, 24, 27, 43, 70].

The preceding assertion needs considerable elaboration to be fully or minimally understood. In such a case we could not do better than use a generic example which happens to be the very case we are dealing with here. In essence and in a nutshell, without going into the maize of abstract mathematical arguments characteristic for transfinite se theory, measure theory and the continuum hypothesis the answer is that we will be replacing Borel resummation and differentiation by what is for physicists, more familiar Weyl scaling [12, 44, 67].

\subsection{The Main Sequence of E-infinity Weyl Scaling}

As known from E-infinity, differentiation and integration are replaced by down scaling and up scaling respectively [16-18]. This is somewhat similar to the replacement of differentiation by a Poisson Bracket and integration with Dixmier trace in A. Connes' non-commutative geometry [110]. Let us start from the theoretically exact value of the inverse fine structure constant:

$\bar{\alpha}_{\mathbf{o}}=(20)(1 / \phi)^{4}=137+\mathrm{k}_{\mathrm{o}}$

For a Cooper pair, we have $\bar{\alpha}_{0} / 2$ where $k_{o}=\phi^{5}\left(1-\phi^{5}\right)$ and $\phi=2 /(1+\sqrt{5})$. Scaling $\bar{\alpha}_{0} / 2$ down using the fundamental scaling factor $\phi[16]$ one finds the following 6 "quasi-differentiated" sequence [30-33]:

$$
\begin{aligned}
\left(\bar{\alpha}_{\mathrm{o}} / 2\right)(\phi)^{\mathrm{n}} \stackrel{\mathrm{n}=1}{\longrightarrow} 42+2 \mathrm{k} \\
\stackrel{\mathrm{n}=2}{\longrightarrow} 26+\mathrm{k} \\
\stackrel{\mathrm{n}=3}{\longrightarrow} 16+\mathrm{k} \\
\stackrel{\mathrm{n}=4}{\longrightarrow} 10 \\
\stackrel{\mathrm{n}=5}{\longrightarrow} 6+\mathrm{k} \\
\stackrel{\mathrm{n}=6}{\longrightarrow} 4-\mathrm{k}
\end{aligned}
$$

Notice we always have an integer plus or minus a multiple of $\mathrm{k}=2 \phi^{5}$ used earlier on to regulate the singularities in 'tHooft-Veltman-Wilson method. Thus setting $\mathrm{k}=0$ we retrieve the Heterotic superstring dimensional hierarchy in full plus the non-super symmetric grand unification inverse coupling constant $\bar{\alpha}=42+2 \mathrm{k} ; 42$. The reader should assure himself or herself that taking $\bar{\alpha}_{\mathrm{o}}=137 / 2$ without the small irrational number $\mathrm{k}_{\mathrm{o}}=\phi^{5}\left(1-\phi^{5}\right)$ will result in a rather messy numerical chain reaction obscuring the neat result found by simply setting $\mathrm{k}=0$ as we just demonstrated. The preceding result will also encourage us to think seriously about looking at $\mathrm{k}$ and consequently also at $\phi^{5}$ as well as $\mathrm{k}_{\mathrm{o}}=\phi^{5}\left(1-\phi^{5}\right)$ not only as perturbation book keeping devices or simply unnecessary numbers after the comma which should be better rounded away but as physically meaningful objects such as instantons, texture, domain walls and exotic topological defects as well as other objects resulting from the disintegration of the vacuum discussed by many others including the present one many years ago [66].

\subsection{Physical-Topological Interpretation of 'tHooft Or- der Parameter $\varepsilon=k=2 \phi^{5}$}

The disintegration of the simplectic vacuum was studied in several earlier publications in connection with paradoxical decomposition and fractal Cantorian spacetime as a source of exotic particles. Two new particles were identified on this occasion with conjectured mass of $26 \mathrm{Mev}$ and $42 \mathrm{Mev}$ matching those reported in scattered experimental research conducted in Darmstadt as well as Cairo as reported by $\mathrm{L}$. Nottale in his classical book. The most important finding 
from that period however, at least as far as the present work is concerned, is the conjectured exotic quasi particle with a mass [142, 143]:

$\mathrm{M}(\mathrm{K})=0.18033989 \mathrm{Mev}$

as reported by the present Author in Ref. . In that particular paper we were also concerned with the phenomena of anomalous positron production as well as gravitational instantons again within the basically fractal setting of a symplectic geometry. The incredible coincidence of $\mathrm{M}(\mathrm{k})=2 \phi^{5}$ gauged in $\mathrm{Mev}$ is of course no coincidence because even ten years ago [21] we already felt the logical necessity and aesthetic attraction in the sense of Dirac looking at $\mathrm{K}$ not only as a geometrical object but as a real physical "quasi" particle. This we elaborate next.

\subsection{Fractal Logic and the Mass Spectrum of the Standard Model [18]}

The fuzzy logic related notion of fractal counting of quantum particles which is based on fractal logic was introduced some time ago in connection with the SU(3) SU(2) $\mathrm{U}(1)$ standard model of elementary high energy particles. There it was shown how the three particles of SU(2), namely the experimentally found $\mathrm{W}^{+}, \mathrm{W}^{-}$and $Z^{\circ}$ should not be counted as 3 but as 2.88543824 . The physical meaning of this initially strange proposal becomes more understandable when we see the entire spectrum of all the classical 12 particles. These 12 particles are in fact 14 particles when counted correctly and have the fractal number weight of only [18]:

$$
\begin{aligned}
12-2 \phi^{4} & =\sqrt{\bar{\alpha}_{0}}=11.70820393 \\
& =\frac{\bar{\alpha}_{0}-20}{10}
\end{aligned}
$$

where $\bar{\alpha}_{o}=137.082039325$ is the E-infinity exact inverse value of the electromagnetic fine structure constant and $\phi^{4}$ is the Hardy-Unruh self entanglement. Consequently putting the 2.88543824 under our transfinite-fractal magnifying glass we see immediately that it is 16 times our dimensional regularization $\mathrm{k}=\phi^{3}\left(1-\phi^{3}\right)$, i.e. that used in our interpretation of 'tHooft method where $\mathrm{D}=4-\varepsilon$ and $\varepsilon=\mathrm{k}=2 \phi^{5}$. Consequently we have a highly consistent theory linking in the 16 bosonic dimensions of Heterotic superstring theory with a fractal number of generators of a fuzzy version of the electroweak Lie symmetry group SU(2). We speculate on passing that there may be a link here between anomalous positron production in ultra strong magnetic fields and to the disintegration of $\mathrm{W}^{+}, \mathrm{W}^{-}$and $\mathrm{Z}^{\circ}$ into 16 exotic quasi particles. In this respect it is wise to ponder very deeply the unexpected result of fractal "fuzzy" logic [18] as applied to the 12 gauge bosons of the classical standard model where it was revealed in that the 12 particles are really 14 particles with fractal weight equal 11.7082039325 particles! In particular two particles with the topological charge or weight $16 \mathrm{k}$ $+\mathrm{k}^{2}$ turn out to be equal $3-\mathrm{k}_{\mathrm{o}}$ as an elementary computation easily reveal which is a fractal spatial dimension found from some fundamental equations.
15.7. Dark Energy Density from the Relative Volume of n-Dimensional Spheres [155-157]

A stunning simple approximation for $\mathrm{E}(\mathrm{D})$ could surprisingly be obtained from an elementary computation using nothing more than the relativity volume of 4-D spheres to that of 5-D spheres. Thus from $\operatorname{vol}(\mathrm{n}=4)=4.938$ and $\operatorname{vol}(5)=5.237$ one finds that $\gamma(D)=4.938 / 5.237 \simeq 94 \%$ therefore $E(D) \simeq(21 / 22) \mathrm{mc}^{2}$

\subsection{Intermediate Conclusions}

With the benefit of hindsight we see that the present result, i.e. the existence of an exotic quasi particle $M(k)=2 \phi^{5}$ where $\phi^{5}$ is Hardy's generic value for the entanglement of two quantum particles should have been expected all along and not only after realizing that 'tHooft-Veltman-Wilson

$\mathrm{D}=4-\varepsilon$ spacetime is essentially a fractal spacetime with non-classical Cosserat-like hyperbolic point set geometry. For instance we have known for decades that the arithmetic mean of the sum of the masses of the charged and the neutral $\pi$-meson is to a high accuracy equal to the magnitude of the inverse electromagnetic fine structure constant gauged in Mev [21]. Similar observations were made with regard to $\mathrm{K}$-meson, the proton as well as the famous empirical relation between the mass of the electron and that of the proton and neutron.

In view of all the aforementioned, we cannot hesitate to express our strong view that quantum spacetime is a Cantorian fractal manifold and that without this fact, dimensional regularization could not be applied in the way it is applied and would not have given the right answer to the problem at hand as it did and in full agreement with measurements and observations.

It is remarkable that the preceding Cantorian WeylNottale scale relativity fits seamlessly into the 'tHooftVeltman dimensional regularization scheme $[119,120]$. There we use $4-\mathrm{D}=\in$ to overcome divergence and here we just set $\in=\mathrm{k}=0.18033989$ to account for the topological entanglement due to Hardy's quantum entanglement $\mathrm{P}($ Hardy $)=\phi^{5}=\mathrm{k} / 2$ where $\phi=2 /(1+\sqrt{5})$ [34-36]. It is thus not difficult to see that $\mathrm{E}=\mathrm{mc}^{2}$ must be scaled to (E) $\left(\frac{4-\mathrm{k}}{4}\right)$ to account for uncorrelated parts of the energy. For the correlated parts of the energy, which is the ordinary measurable energy we just need to take the Legendre transform, i.e. the complimentary energy of the uncorrelated so called dark energy, namely $1-\left(\frac{4-\mathrm{k}}{4}\right)$. That way we find the dark energy component:

$\mathrm{E}(\mathrm{D})=\left(\mathrm{mc}^{2}\right)\left(\frac{4-\mathrm{k}}{4}\right)=\left(\mathrm{mc}^{2}\right)\left(\frac{21+\mathrm{k}}{22+\mathrm{k}}\right) \cong\left(\mathrm{mc}^{2}\right)(21 / 22)(60)$

and the ordinary energy component: 
$\mathrm{E}(\mathrm{O})=1-\mathrm{E}(\mathrm{D})=\left(\mathrm{mc}^{2}\right)(21+\mathrm{k}) \cong \mathrm{mc}^{2} / 22$

all in full agreement with previous derivations and cosmic observation and analysis [8]. The preceding analysis is effectively saying that $4-\mathrm{k}$ of " $\mathrm{tHooft}$ and Veltman is more than a mathematical trick to extract the correct result and avoid divergence. It is an aspect of physical reality and indicates the fractal nature of spacetime and the role of "gravity" in eliminating some unwanted mathematical problems in the exact renormalization equation of gauge fields.

\section{DISCUSSION AND FINAL CONCLUSIONS}

The preceding short survey and accompanied computation may be seen as a Cosserat-like material spacetime based analysis in the spirit of the analogies discussed in connection with the pinched elastic cylindrical shell (see Fig. 1) [23]. We stress that the failure of Einstein's general relativity to predict dark energy directly could easily be explained via the Cosserat-like theory presented here [23, 24]. However first Einstein and then Cartan were aware of the problem and proposed what became known later on as the Teleparallelism theory [24, 27]. Thus our Cosserat-like theory points essentially in the same general direction as the Teleparallelism relativity theory $[24,27]$. It should not pass unnoticed that $\mathrm{E}$ $=\mathrm{mc}^{2}$ is not simply a final conclusion in the special theory of relativity [24]. It is far more than that [118]. It implies the general theory of relativity and connects it to thermodynamics long before anyone noticed that including Einstein himself. Needles to repeat what W. Rindler stressed in all his writing that $\mathrm{E}=\mathrm{mc}^{2}$ is a leap of faith and a visionary step which does not follow directly from the special relativity only. However this giant leap has paid off and was a risk worth taking. Finally we must express our deep satisfaction bout the robustness of the result $\mathrm{E}=\left(\mathrm{mc}^{2} / 22\right)+\mathrm{mc}^{2}(21 / 22)=$ $\mathrm{mc}^{2}$ which we always reach using virtually any reasonable theory. The inescapable conclusion is the following: If accelerated cosmic expansion is real and it seems that it is real, then Hawking's radiation, Rindler's horizon and Unruh's temperature are also real. In fact the applicability of Cosserat theory to Dirac's equation [93] and the present work shows that we can regard spacetime as tangibly real and then everything will fall into place the right way. Around the year 2006 the Author pondered the question of which theory is more fundamental, relativity or quantum field $[115,116]$. At the time the question seemed to be Goedelian undecidable. However with present understanding the Author tends to believe relativity is much stronger than we or even Einstein himself ever thought it is. This view seems to be correct when we look deeper at the present results connected to pure gravity and 'tHooft-Veltman renormalization $[119,120]$. As well as the far reaching conjuncture that entanglement and dimensional renormalization maybe mediated by two new elementary quasi particles with appropriate name suggestions, namely entangelon and 'tHoofton. In this connection we must again and again remind ourselves that what we call particles are mathematical singularities and that composite and elementary are notions with no absolute meaning in a fractal setting such as our Cantorian-fractal spacetime. Having traveled a truly long journey to come to the present conclusions, we are compelled by scientific honesty to make an unfortunately immodest statement namely that the golden mean based number system and arithmetic behind our transfinite computer constituting an infinite time Cellular Automata is the only way to do constrictive real computations using infinities and divergences at singularities which are perceived by us physically as quasi particles similar to the present entangelon, 'tHoofton and our much earlier proposal regarding a fluction particle $[162,163]$.

In closing our discussion we feel we should mention 'tHooft's strong interest in Cellular Automata as rational quantum mechanics and that our Cantorian-Fractal spacetime proposal amounts to a transfinite Cellular Automata [18, $158,161]$. Last but not least, we draw attention to the related theory of xonic quantum physics developed in France and the USA mainly by Jean-Paul Auffray [164].

\section{CONFLICT OF INTEREST}

The author confirms that this article content has no conflict of interest.

\section{ACKNOWLEDGEMENTS}

The Author is genuinely deeply indebted to two exceptionally rigorous as well as fair and encouraging Referee reports that were more than helpful in producing the present paper.

\section{REFERENCES}

[1] Heisenberg W. Der Teil und das Ganze. Munich: Piper 1969; 2.

[2] Weyl H. Raum - Zeil - Materie. Berlin: Springer 1923.

[3] Hawking S, Penrose R. The nature of space and time. New Jersey: Princeton University Press 1996.

[4] Weibel P, Ord G, Rössler O, Eds. Space-time physics and fractality: Festschrift in honor of Mohamed El Naschie on the occasion of his $60^{\text {th }}$ birthday. Vienna-New York: Springer 2005.

[5] Halpern P. The great beyond. New Jersey: John Wiley 2004.

[6] Ye Fred Y. From chaos to unification: U-Theory vs M-Theory. Chaos Soliton Fract 2009; 42: 89-93.

[7] Marek-Crnjac L. An invitation to El Naschie's theory of Cantorian space-time and dark energy. Int J Astron Astrophys 2013; 3: 46471.

[8] He Ji-Huan, Marek-Crnjac L. The quintessence of El Naschie's theory of fractal relativity and dark energy. Fract Spacetime Noncommutative Geometry Quantum High Energy Phys 2013; 3: 1307.

[9] El Naschie MS. Experimentally based theoretical arguments that unruh's temperature, hawkings's vacuum fluctuation and rindler's wedge are physically real. Am J Mod Phys 2013; 2(6): 357-61.

[10] Persinger M, Koren S. Dimensional analysis of geometric products and the boundary conditions of the universe: implications for quantative value for the latency to display entanglement. Open Astron J 2013; 6: 10-13.

[11] Yang Xiao-Jun, Baleanu D, Zhong Wei-Ping. Approximate solutions for diffusion equations on Cantor space-time. Proc Rom Acad Series A 2013; 14(2): 127-33.

[12] Marek-Crnjac L. Cantorian space-time theory - the physics of empty sets in connection with quantum entanglement and dark energy. Saarbrücken: Lambert Academic Publishing 2013.

[13] El Naschie MS. Transfinite neo-impressionistic reality of quantum space-time. New Adv Phys 2007; 1(2): 111-22. 
[14] Malinowski JJ. Fractal physics theory-foundation. J Mod Phys 2011; 1(2): 133-68.

[15] El Naschie MS. Nash embedding of Witten's M-theory and Hawking-Hartle quantum wave of dark energy. J Mod Phys 2013; 4(10): 1417-28.

[16] El Naschie MS. From Yang-Mills photon in curved space-time to dark energy density. J Quantum Info Sci 2013; 3(4): 121-6.

[17] El Naschie MS. A Rindler-KAM space-time geometry and scaling the Planck scale solves quantum relativity and explains dark energy. Int J Astron Astrophys 2013; 3(4): 483-93.

[18] El Naschie MS. On the need for fractal logic in high energy quantum physics. Int J Mod Nonlinear Theory Appl 2012; 1(3): 84-92.

[19] El Naschie MS. Quantum entanglement as a consequence of a Cantorian micro space-time geometry. J Quantum Info Sci 2011; 1(2): 50-3

[20] El Naschie MS. A resolution of cosmic dark energy via a quantum entanglement relativity theory. J Quantum Info Sci 2013; 3(1): 236.

[21] El Naschie MS. A review of E-infinity and the mass spectrum of high energy particle physics. Chaos Soliton Fract 2004; 19(1): 20936.

[22] Helal MA, Marek-Crnjac L, He Ji-Huan. The three page guide to the most important results of M.S. El Naschie's research in Einfinity and quantum physics and cosmology. Open J Microphys 2013; 3(4): 141-5.

[23] El Naschie MS. Quantum gravity and dark energy using fractal Planck scaling. J Mod Phys 2013; 4(11A): 31-8.

[24] Penrose R. The road to reality. London: Jonathan Cape 2004.

[25] El Naschie MS. Stress, stability and chaos in structural engineering: an energy approach. London-Tokyo: McGraw Hill Int. Editions Civil Eng Series 1990.

[26] He Ji-Huan, Marek-Crnjac L. Mohamed El Naschie's revision of Albert Einstein's E $=\mathrm{mc}^{2}$ : A definite resolution of the mystery of the missing dark energy of the cosmos. Int J Mod Nonlinear Sci Appl 2013; 2(1): 55-9.

[27] El Naschie MS. A unified Newtonian-relativistic quantum resolution of the supposedly missing dark energy of the cosmos and the constancy of the speed of light. Int J Mod Nonlinear Sci Appl 2013; 2(1): 43-54.

[28] Marek-Crnjac L. Chaotic fractal tiling for the missing dark energy and Veneziano model. Appl Math 2013; 4(11B): 22-9.

[29] Susskind L, Lidesay J. The holographic universe: an introduction to black holes, information and the string theory revolution. Singapore: World Scientific 2005.

[30] Ellis GFR, Williams RM. Flat and curved space-time. Oxford: Oxford University Press 2000.

[31] Bronshtein IN, Semendyayev KA. Handbook of mathematics. New York: Van Nostrand Reinhold Company 1985.

[32] Padmanabhan T. Dark Energy: The cosmological challenge of the millennium 2004. arXiv: astro-ph/0411044V1.

[33] Padmanabhan T. Gravity from space-time thermodynamics. Astrophys Space Sci 2003; 285(2): 407-17.

[34] El Naschie MS. Topological-geometrical and physical interpretation of the dark energy of the cosmos as a 'halo' energy of the Schrödinger quantum wave. J Mod Phys 2013; 4(5): 591-6.

[35] El Naschie MS. What is the missing dark energy in a nutshell and the Hawking-Hartle quantum wave collapse. Int $\mathbf{J}$ Astron Astrophys 2013; 3(3): 205-11.

[36] El Naschie MS, Helal A. Dark energy explained via the HawkingHartle quantum wave and the topology of cosmic crystallography. Int J Astron Astrophys 2013; 3(3): 318-43.

[37] El Naschie MS. Adic unification of the fundamental forces and the standard model. Chaos Soliton Fract 2008; 38(4): 1011-12.

[38] El Naschie MS. Advanced prerequisites for E-infinity. Chaos Soliton Fract 2006; 30(3): 636-41.

[39] El Naschie MS. Quantum gravity from descriptive set theory. Chaos Soliton Fract 2004; 19(5): 1339-44.

[40] El Naschie MS. P-Adic analysis and the transfinite E8 exceptional Lie symmetry group unification. Chaos Soliton Fract 2008; 38(3): 612-14.

[41] El Naschie MS. A review of application and results of E-infinity theory. Int J Nonlinear Sci Num Simul 2007; 8(1): 11-20.
[42] Vladimirov V, Valovich I, Zelenov E. P-Adic analysis and mathematical physics. Singapore: World Scientific 1994.

[43] Khrennikov A. Non-archimedean analysis: Quantum paradoxes, dynamical systems and biological methods. Dordrecht-London: Kluwer Academic Publishers 1997.

[44] El Naschie MS. The theory of cantorian space-time and high energy particle physics (an informal review). Chaos Soliton Fract 2009; 41(5): 2635-46.

[45] El Naschie MS. Elementary prerequisites for E-infinity (Recommended background readings in nonlinear dynamics, geometry and topology). Chaos Soliton Fract 2006; 30(3): 579-605.

[46] El Naschie MS. The concepts of E-infinity: An elementary introduction to the Cantorian-fractal theory of quantum physics. Chaos Soliton Fract 2004; 22(2): 495-511.

[47] Ruiz-Lapuente Pilar, Ed. Dark energy. Cambridge: Cambridge University Press 2010.

[48] Perkins D. Particle astrophysics. $2^{\text {nd }}$ ed. Oxford: Oxford University Press 2009.

[49] Amendola L, Tsujikawa S. Dark energy-theory and observations. Cambridge: Cambridge University Press 2010.

[50] Bahcall J, Pivan T, Weinberg S, Eds. Dark matter in the Universe, $2^{\text {nd }}$ ed. Singapore: World Scientific 2004.

[51] Weinberg S. Cosmology. Oxford: Oxford University Press 2008.

[52] Vilenkin A, Shellard ES. Cosmic strings and other topological defects. Cambridge: Cambridge University Press 1994.

[53] El Naschie MS. Wild topology, hyperbolic geometry and fusion algebra of high energy particle physics. Chaos Soliton Fract 2002; 13(9): 1935-45.

[54] El Naschie MS. Quantum loops, wild topology and fat Cantor sets in transfinite high energy physics. Chaos Soliton Fract 2002; 13(5): 1167-74.

[55] Tao Yong. The validity of dimensional regularization method on fractal space-time. J Appl Math 2013; arXiv: http://dx.doi.org/10.1155/2013/308691.

[56] Marek-Crnjac L. A Feynman path integral-like method for deriving the four dimensionality of space-time from first principles. Chaos Soliton Fract 2009; 41(5): 2471-3.

[57] El Naschie MS. Determining the temperature of the microwave background radiation from the topology and geometry of spacetime. Chaos Soliton Fract 2002; 14(7): 1121-6.

[58] El Naschie MS. A note on quantum gravity and Cantorian spacetime. Chaos Soliton Fract 1997; 8(1): 131-3.

[59] El Naschie MS. Superstrings, knots and noncommutative geometry in E-infinity space. Int J Theor Phys 1998; 37(12): 2935-51.

[60] Lehmann T. Formänenderung eines klassischen kontinuums in vierdimensionaler darstellung. Applied Mechanics. Springer Verlag H. Görtler, Ed. Heidelberg: Springer 1966; 376-82.

[61] Mukhamedov AM. E-infinity as a fibre bundle and its theormodynamics. Chaos Soliton Fract 2007; 33(3): 717-24.

[62] El Naschie MS. Die Ableitung einer kosistenten schalentheorie aus dem dreidimensionalen Kontinuum. Aust Eng J 1979; 22(9): 33944.

[63] El Naschie MS. Curvature, lagrangian and holonomy of cantorianfractal space-time. Chaos Soliton Fract 2009; 41(4): 2163-7.

[64] El Naschie MS. An irreducibly simple derivation of the Hausdorff dimension of space-time. Chaos Soliton Fract 2009; 41(5): 1902-4.

[65] El Naschie MS. Quantum groups and Hamiltonian sets on a nuclear space-time Cantorian manifold. Chaos Soliton Fract 1999; 7(11): 1251-6.

[66] El Naschie MS. The symplectic vacuum, exotic quasi particles and gravitational instantons. Chaos Soliton Fract 2004; 22(1): 1-11.

[67] El Naschie MS. Transfinite harmonization by taking the dissonance out of the quantum field symphony. Chaos Soliton Fract 2008; 36(4): 781-6.

[68] Vrobel S. Fractal time. Singapore: World Scientific 2011.

[69] El Naschie MS. The minus one connection of relativity, quantum mechanics and set theory. Fract Spacetime Noncommutative Geometry High Energy Phys 2012; 2(2): 131-4.

[70] El Naschie MS, Olsen S. When zero is equal to one. A set theoretical resolution of quantum paradoxes. Fract Space-time Noncommutative Geometry High Energy Phys 2011; 1(1): 11-24. 
[71] El Naschie MS. The quantum gravity Immirzi parameter - A general physical and topological interpretation. Gravitation Cosmol 2013; 19(3): 151-5.

[72] El Naschie MS. Determining the missing dark energy of the cosmos from a light cone exact relativistic analysis. J Phys 2013; 2(2): 18-23.

[73] Adams CC. The knot book. New York: H. Freeman 1994; pp. 2446.

[74] Pathvia RK. The universe as a black hole. Nature 1972; 240: 298-9.

[75] Nesteruk A. Physics in christianity. Encyclopedia Sci Relig 2013; 3: 1718-29.

[76] Watson A, Reid S, Johnson W, Thomas S. Large deformations of thin-walled circular tubes under transverse loading - II. Int J Mech Sci 1976; 18: 387-97.

[77] Marek-Crnjac L. Quantum gravity in cantorian space-time. Rodrigo-Soberio, Ed. INTECH Publishing 2012; pp. 87-100. Available from: www.intechopen.com.

[78] Cosserat E, Cosserat F. Théorie des Corps déformables. Paris: Hermann 1909.

[79] El Naschie MS. The hyperbolic extension of Sigalotti-HeniSharifzadeh's golden triangle of special theory of relativity and the nature of dark energy. J Mod Phys 2013; 4(3): 354-6.

[80] Linder E. Dark energy in a "Scholarpedia blog". The peer-reviewed open access Encyclopedia 2008; 3(2): 4900. Doi: 10.4249/scholarpeadia.4900.

[81] Sahni V. Theoretical models of dark energy. Chaos Soliton Fract 2003; 16(4): 527-37.

[82] Finn R. Capillary surface interface. Am Math Soc 1999; 46(7): 770-81.

[83] Dierkes V, Hildebrandt S. Minimal surfaces I. Berlin: Springer Verlag 1992.

[84] El Naschie MS. Using Witten's five brane theory and the holographic principle to derive the value of the electromagnetic fine structure constant $\bar{\alpha}_{\mathrm{o}}=1 / 137$. Chaos Soliton Fract 2008; 38(4): 1051-3.

[85] El Naschie MS. Fuzzy knot theory interpretation of Yang-Mills instantons and itten's 5 brane model. Chaos Soliton Fract 2008; 38(5): 1349-54.

[86] El Naschie MS, Helal MA. Dark energy explained via the Hawking-Hartle quantum wave and the topology of cosmic crystallography. Int J Astron Astrophys 2013; 3(3): 318-43.

[87] El Naschie MS. Dark energy explained via quantum field theory in curved space-time. J Mod Phys App 2014; 2: 1-7.

[88] El Naschie MS. The missing dark energy of the cosmos from light cone topological velocity and scaling the Planck scale. Open J Microphys 2013; 3(3): 64-70.

[89] El Naschie MS. The measure concentration of convex geometry in a quasi Banach spacetime behind the supposedly missing dark energy of the cosmos. Am J Astron Astrophys 2014; 2(6): 72-7.

[90] Hehl F. Space-time as generalized cosserat continuum. In "Mechanics of generalized continua", Kronev E, Ed. Berlin: Springer Verlag 1968; pp.347-9.

[91] Chao-Qiang G, Chung-Chi L, Saridakis EN, Wu Yi-Peng. Teleparallel Dark Energy 2001. ArXiv: 1109.1092v2 [hep-th]

[92] Hehl F and Obukhov Y. Elie Cartan's torsion in geometry and in field theory: An essay. Annales de la foundation Louis de Broglie 2007. arXiv: 0711.1535v1[gr-9c].

[93] Burnett J, Chervova O, Vassiliev D. Dirac equation as a special case of Cosserat elasticity 2008. arXiv: 0812.3948v1[gr-9c].

[94] El Naschie MS. SU(5) grand unification in a transfinite form. Chaos Soliton Fract 2007; 32(2): 370-4.

[95] El Naschie MS. SO(10) grand unification in a fuzzy setting. Chaos Soliton Fract 2007; 32(3): 958-61.

[96] El Naschie MS. High energy physics and the standard model from exceptional Lie groups. Chaos Soliton Fract 2008; 36(1): 1-17.

[97] El Naschie MS. Symmetry groups pre-requisite for E-infinity in high energy physics. Chaos Soliton Fract 2008; 35(1): 202-11.

[98] El Naschie MS. Notes on exceptional lie symmetry groups hierarchy and possible implications for E-infinity high energy physics. Chaos Soliton Fract 2008; 35(1): 69-70.

[99] Duff MJ. The World in eleven dimensions. Bristol: Institute of Physics Publications 1999.
[100] Penrose R. The road to reality. London: Jonathan Cape 2004.

[101] Cartan Elie. Espaces á connexion affine, projective et conforme. Acta Math 1926; 48: 4-42.

[102] Czajko J. Elie cartan and pan-geometry of multispatial hyperspace. Chaos Soliton Fract 2004; 19(3): 479-502.

[103] Kaku M. Introduction to superstrings and M-Theory. New York: Springer Verlag 1999.

[104] Becker K, Becker M, Schwarz JH. String theory and M-Theory. Cambridge: Cambridge University Press 2007.

[105] El Naschie MS. On the Witten-Duff five branes model together with knots theory and E8E8 superstrings in a single fractal spacetime theory. Chaos Soliton Fract 2009; 41(4): 2016-21.

[106] Rezzalla L, Zanotti Olindo. Relativistic hydrodynamics. Oxford: Oxford University Press 2013.

[107] Distler J, Kawai H. Hausdorff dimension of continuous Polyakov's random surface. Int J Mod Phys A 1990; 5(6): 1093.

[108] Duff M, von Nieuwenhuizen P. Quantum inequivalence of different field representation. Phys Lett B 1980; 94(2): 179-82.

[109] Marek-Crnjac L. From Arthur Cayley via Felix Klein, Sophus Lie, Wilhelm Killing, Ellie Cartan, Emmy Noether superstrings to cantorian spacetime. Chaos Soliton Fract 2006; 37(5): 1279-88.

[110] Connes A. Noncommutative geometry. San Diego: Academic Press 1994.

[111] El Naschie MS. The logic of interdisciplinary research. Chaos Soliton Fract 1997; 8(9): vii-x

[112] El Naschie MS. The initial post buckling of an extensional ring under external pressure. Int J Mech Sci 1975; 17(6): 387-8.

[113] El Naschie MS. The role of formulation in elastic buckling. PhD Thesis, Civil Eng. Dept. University College. University of London: 1974.

[114] El Naschie MS. Physically nonlinear warping torsion of thin walled beams. Master Thesis, Dept. of Mechanics, University of Hannover Germany: (No. A/1573)16.40 1969. (in German).

[115] El Naschie MS. Is gravity less fundamental than elementary particles theory. Chaos Soliton Fract 2006; 29(4): 803-7.

[116] El Naschie MS. Is Einstein's general field equation more fundamental than quantum field and particle physics? Chaos Soliton Fract 2006; 30(3): 525-31.

[117] Polchinski J. String theory, Vol. I and Vol. II. Cambridge: Cambridge University Press 1998.

[118] Rindler W. Relativity, special, general and cosmology. $2^{\text {nd }}$ ed. Oxford: Oxford University Press 2006.

[119] t'Hooft G. A Confrontation with Infinity. In: Sidharth B, Altaisky M, Eds. Frontiers of Fundamental Physics 4. New York: KluwerPlenum 2001; pp.1-12.

[120] El Naschie MS. 'tHooft's dimensional regularization implies transfinite Heterotic string theory and dimensional transmutation. In: Sidharth B, Altaisky M, Eds. Frontiers of Fundamental Physics 4. New York: Kluwer-Plenum 2001; pp.81-6.

[121] El Naschie MS. The Cantorian gravity coupling constant is $\bar{\alpha}_{g s}=$ 1/26.18033989. In: B Sidharth, M Altaisky, Eds. Frontiers of Fundamental Physics 4. New York: Kluwer-Plenum 2001; pp. 87-96.

[122] 'tHooft G. Dimensional regularization and the renormalization group. Nucl Phys B 1973; 61: 455-68.

[123] 'tHooft G, Veltman M. Regularization and renormalization of gauge fields. Nucl Phys B 1972; 44: 189-213.

[124] Wilson KG, Kogul J. The renormalization group and the E expansion. Phys Rep 1974; 12(2): 75-199.

[125] El Naschie MS. Cosmic dark energy from 'tHooft's dimensional regularization and Witten's topological quantum field pure gravity. J Quantum Info Sci 2014; 4: 83-91.

[126] El Naschie MS. Asymptotically safe pure gravity as the source of dark energy of the vacuum. Astrophys Space Sci 2014; 2(1): 12-5.

[127] Wilson KG. Critical phenomena in 3.99 dimensions. Physica 1974; 73: 119-28.

[128] El Naschie MS. Cosserat-Cartan modification of Einstein-Riemann relativity and cosmic dark energy density. Am J Mod Phys 2014; 3(2): 82-7.

[129] Wilson KG, Fisher ME. Critical exponents in 3.99 dimensions. Phys Rev Lett 1972, 28: 240-3. 
[130] El Naschie MS. On 'tHooft dimensional regularization in E-infinity space (with letter from R. Feynman to G. Ord dated 1982). Chaos Soliton Fract 2001; 30(5): 855-8.

[131] Brezin E. Wilson renormalization group: a paradigmatic shift. 2014. arXiv: 1402.34337VI[physics.hist-ph].

[132] Polyakov AM. Conformal symmetry of critical fluctuation. ZHETF Pis Red 1970; 12: p. 538

[133] El Naschie MS. 'tHooft ultimate building blocks and space-time as an infinite dimensional set of transfinite discrete points. Chaos Soliton Fract 2005; 25(3): 521-4.

[134] Marek-Crnjac L. Partially ordered sets, transfinite topology and the dimension of Cantorian fractal space-time. Chaos Soliton Fract 2009; 42(3): 1796-9.

[135] Zhong T. From the numerics of dynamics to the dynamics of numeric and vice versa in high energy particle physics. Chaos Soliton Fract 2009; 42(3): 1780-3.

[136] Graham L, Kantor J. Naming infinity. USA: Harvard University Press 2009.

[137] El Naschie MS. Casimir-like energy as a doube Eigenvalues of quanumly entangled system leading to the missing dark energy density of the cosmos. Int J High Energy Phys 2014; 1(5): 55-63.

[138] El Naschie MS. E-infinity High Energy communications Nos. 7190. El Naschie Watch - Genuine Scientific Blog for E-infinity, noncommutative geometry, fractal space-time, innovative geometrical and number theoretical methods in high energy physics and quantum gravity. http://www.elnaschiewatch.com. (From 18 January 2011 to 16 December 2012).

[139] Ho Mae-Wan. The story of phi, part 1. Watching the daises grow, part 2. Golden music of the brain, part 3. Golden cycles and organic space-time, part 4. Golden geometry of e-infinity fractal spacetime, part 5. J Inst Sci Soc 2014; www.i-sis.org.uk.

[140] Ho Mae-Wan. ISIS Report: E-infinity space-time, quantum paradoxes and quantum gravity. J Inst Sci Soc Reports Nos. 03/03/14 to 07/04/14.

[141] May P. E-infinity spaces and E-infinity ring spectra. Lecture notes in mathematics. Berlin: Springer Verlag 1977.

[142] Naschie MS. Topological defects in the symplectic vacuum anomalous positron production and gravitational instantons. Int $\mathrm{J}$ Mod Phys E 2004; 835.

[143] El Naschie MS. New elementary Particles As a possible product of the disintegration of the symplectic vacuum. Chaos Soliton Fract 2004; 20(4): 905-13.

[144] Shalaby AM. The fractal self-similar Borel algorithm for the effective potential of the scalar field theory in one time plus one space dimensions. Chaos Soliton Fract 2007; 34(3): 709-16.

[145] El Naschie MS. Rindler space derivation of dark energy. J Mod Phys Appl 2014; ID 6.

[146] El Naschie MS. Kaluza-Klein unification - some possible extensions. Chaos Soliton Fract 2008; 37(1): 16-22.
[147] El Naschie MS. Dark energy from Kaluza-Klein space-time and Noether's theorem via Lagrangian multiplier method. J Mod Phys 2013; 4: 757-60.

[148] Marek-Crnjac L, El Naschie MS, He Ji-Huan. Chaotic fractals at the relativistic quantum physics and cosmology. Int J Mod Nonlinear Theor Appl 2013; 2: 78-88.

[149] El Naschie MS. Pinched material Einstein space-time produces accelerated cosmic expansion. Int J Astron Astrophys 2014; 4: 8090.

[150] El Naschie MS. From classical gauge theory back to Weyl scaling via E-infinity space-time. Chaos Soliton Fract 2008; 38(4): 980-5.

[151] Nottale L. Scale relativity and fractal space-time. London: Imperial College Press 2011.

[152] El Naschie MS. On the fundamental equations of the constants of nature. Chaos Soliton Fract 2008; 35: 320-3.

[153] El Naschie MS. To dark energy theory from a Cosserat-like model of space-time. Prob Nonlinear Anal Engin Sys 2014; 20(1): 41.

[154] El Naschie MS, Marek-Crnjac L, Helal MA, He Ji-Huan. A topological magueijo-smolin varying speed of light theory, the accelerated cosmic expansion and the dark energy of pure gravity. Appl Math 2014; 5(12): 1780-90.

[155] El Naschie MS. On spaces with 26 dimensions, Chaos Soliton Fract 2000; 11(8): 1335-8.

[156] El Naschie MS. Cobe satellite measurement, hyperspheres, superstrings and the dimension of space-time. Chaos Soliton Fract 1998; 9(8): 1445-71.

[157] El Naschie MS. The mean sphere $\langle S\rangle$ as a three manifold in $\mathrm{R}^{4}$. Chaos Soliton Fract 1998; 9(9): 1631-5.

[158] Svozil K. Computational universes, Chaos Soliton Fract 2005; 25(4): 845-59.

[159] Givors F, Lafitte G, Ollinger N. Infinite time cellular automata: A real computation model. Journees Automates Cellulaires 2010; 111-20.

[160] Pavlos GP. Complexity theory and physical unification: from microscopic to macroscopic level. In: Dimotikalis I, Skiadas C, Eds. Chaos Theory: Modeling, simulation and applications. Singapore: World Scientific Publishing 2011; pp. 297-308.

[161] El Naschie MS. On the universality class of all universality classes and E-infinity space-time physics. Chaos Soliton Fract 2007; 32(3): 927-36.

[162] El Naschie MS. Towards a geometrical theory for the unification of all fundamental forces. Chaos Soliton Fract 2000; 11(9): 1459-69.

[163] El Naschie MS. Scale relativity in Cantorian E-Infinity space-time. Chaos Soliton Fract 2000; 11(14): 2391-6.

[164] Auffray JP. E-infinity dualities, discontinuous space-time, xonic quantum physics and the decisive experiment. J Mod Phys 2014; 5: 1427-36.

(C) Mohamed S. El Naschie; Licensee Bentham Open.

This is an open access article licensed under the terms of the Creative Commons Attribution Non-Commercial License (http://creativecommons.org/licenses/by-nc/3.0/) which permits unrestricted, non-commercial use, distribution and reproduction in any medium, provided the work is properly cited. 